\title{
Small-Distance-Behaviour Analysis and Wilson Expansions
}

\author{
K. SYMANZIK \\ Deutsches Elektronen-Synchrotron DESY, Hamburg
}

Received July 9, 1971

\begin{abstract}
A previously described method to obtain the asymptotic forms of vertex functions at large momenta is, with the help of Wilson operator product expansion formulas, extended to momenta where the vertex functions of the zero-mass theory underlying the asymptotic forms are infrared singular. To obtain from asymptotic forms information on asymptotic behaviour requires assumptions on the behaviour of the zero-mass theory in the limit of infinite dilatation. One particular set of assumptions is discussed and found to pass a simple consistency test; this set of assumptions leads to power laws, or slight modifications thereof, with coupling-constant-independent exponents. The detailed discussion is given for the $\phi^{4}$ model.
\end{abstract}

\section{Introduction}

Of great interest in quantum field theory is the asymptotic behaviour of vertex functions (VFs) (i.e., the amputated one-particle irreducible parts of Green's functions $)^{1} \Gamma\left(p_{1} \ldots p_{2 n}\right), \Sigma p=0$, as the momenta become large. We formalize this question by investigating

$$
f(\lambda)=\Gamma\left(\left(\lambda p_{1}+r_{1}\right) \ldots\left(\lambda p_{2 n}+r_{2 n}\right)\right), \Sigma p=\Sigma r=0,
$$

for large $\lambda$. In perturbation theory one obtains [1-3], formally,

$$
f(\lambda)=\lambda^{2 \alpha} \sum_{k=0}^{\infty} \sum_{l=0}^{\infty} f_{k l} \lambda^{-k}(\ln \lambda)^{l}
$$

where the even integer $2 \alpha$ is Weinberg's [1] asymptotic exponent and depends on $n$ and the momenta $p_{1} \ldots p_{2 n}$, and each $f_{k l}$ is an infinite power series in $g$. Keeping in (0.2) only the $k=0$ part defines the asymptotic form (AF) of $f(\lambda)$; keeping also the parts with $k \leqq m$ defines the AF of $m^{\text {th }}$ degree ${ }^{2}$.

${ }^{1}$ For conciseness, we write formulas for the $g \phi^{4}$ theory, the considerations of this introduction and in essence of the whole paper being valid, however, for all renormalizable field theories.

${ }^{2}$ If we use the term $\mathrm{AF}$ without specifying the degree, we mean the $\mathrm{AF}$ of zeroth degree.

4 Commun. math. Phys, Vol. 23 
That the AF of any finite, in particular of the zeroth, degree approximates $f(\lambda)$ at large $\lambda$ in the obvious sense is an assumption always made but impossible to test without performing all the relevant summations in (0.2), for which no technique is available yet.

The renormalization group approach $[4,5]$ reveals certain general relations between the $f_{k l}$. These relations allow e.g. to calculate easily the term in $f_{0 l}$ of lowest appearing order $(n+l$ if $n \geqq 3)$ in $g$ (the "leading logarithm"), of next to lowest order, etc. The sums of those terms yield, however, expressions (see Refs. $[5,6,7]$ ) that make no sense for $\lambda$ sufficiently large. Thus, though one may hope that it is not necessary to go beyond $k=0$, he must attempt to deal with the corresponding complete $l$-sum, i.e., with the precise AF. The renormalization group technique indeed leads to certain formal properties of that AF.

Recently [8] we have developped a new tool to study the AFs of VFs, namely, insertion of a (possibly, generalized [9]) mass term into the Lagrangian, the infinitesimal operation thereto resulting in certain simple inhomogeneous partial differential equations (PDEs) ((I.12) below, abbreviated $O \not \Gamma=\Delta \Gamma$ ) being obeyed by the VFs. For any $\Gamma$, that solution $\Gamma_{a s}$ of $\mathcal{O} p \Gamma_{a s}=0$ which approaches $\Gamma$ for small mass is the AF of $\Gamma$, and actually is the VF of a certain zero-mass theory [10].

$\Gamma_{a s}$ has, due to masslessness of the particles ${ }^{3}$, infrared (UR) singularities at certain momenta at which $\Gamma$ itself is not singular. At these momenta, which we call exceptional momenta [10], the correct AF of $\Gamma$, denoted by $\Gamma_{\underline{a s}}$, is not obtained by restriction of $\Gamma_{a s}$, and $\mathcal{O} p \Gamma_{\underline{a s}} \neq 0$ due to the nonnegligibility of $\Delta \Gamma$ relative to $\Gamma$ at large momenta (or, at fixed momenta but small mass). Furthermore, while for nonexceptional momenta $p_{i}$ in (0.1) the $\mathrm{AF}\left(k=0\right.$ in (0.2)) $\Gamma_{a s}$ is independent of the $r_{i}$, this does not hold ${ }^{4}$, at exceptional momenta, for the $\Gamma_{\underline{a} s}$.

In this paper we show, by treating a number of examples, how at exceptional momenta ${ }^{5}$ the correct $\mathrm{AF}$ of $\Gamma, \Gamma_{\underline{a} \underline{s}}$, is obtained through analysis of $\Delta \Gamma$. If for $\Delta \Gamma$, which then has the same asymptotic exponent $2 \alpha$ in $(0.1)$ as $\Gamma$, its $\mathrm{AF} \Delta \Gamma_{\underline{a s}}$ is inserted into the PDE, $\mathcal{O} \not \Gamma_{\underline{a} s}=\Delta \Gamma_{\underline{a} \underline{s}}$ is the correct equation, whose solution approaching $\Gamma$ for small mass being

${ }^{3}$ The possibility of particle interpretation of the zero-mass theory is, however, special to the $\phi^{4}$ model and is absent in QED [10].

${ }^{4}$ The AFs of higher degree always depend on the $r_{i}$; if all $r_{l}$ are zero, the $f_{k l}$ with $k$ odd vanish.

${ }^{5}$ In the analysis of short-distance behaviour of operator products using Wilson expansions $[9,11-13]$ one is led for the singular $c$-number coefficient functions to precisely the problem of behaviour of simple VFs at exceptional momenta, see e.g. the examples in Section III.4. This also holds for the short-distance behaviour of products of currents in theories with internal symmetry, to whose Green's functions involving current operators the techniques of this paper are easily extensible (cp., e.g., Ref. [14]). 
the sought correct AF of $\Gamma$. At occasion, the PDE turns out actually to be homogeneous, $\Delta \Gamma_{\underline{a} \underline{s}}$ being proportional to $\Gamma_{\underline{a} s}$.

The tool to obtain $\Delta \Gamma_{\underline{a s}}$ are Wilson's operator product expansions $[9,11]$, more precisely, certain generalizations of formulas derived in all order of perturbation theory by Brandt [12] and Zimmermann [13]. For those formulas we shall use we sketch an elementary proof in Appendix A.

The renormalization group technique should yield, in the examples we treat, the same result as we obtain. However, the technique described here has advantages. It is simpler ${ }^{6}$ and, since it relies directly on power counting, more transparent, and is the more economical one since it delegates an important, and apparently the largest possible, part of the analysis to formally rigorous, nonasymptotic, considerations.

The relation to zero-mass theories behind the AFs is here not less close than it is in the renormalization group approach, and actually more explicit. If in (0.1) the $r_{i}$ are zero, the AF of second degree is easily obtained, for nonexceptional momenta $p_{i}$, by solving the PDE with $\Delta \Gamma$ replaced by its AF, $\Delta \Gamma_{\underline{a s}}$, which has asymptotic exponent two less than $\Gamma$ itself, and we carry this out for the inverse propagator. In principle, AFs of arbitrarily high degree can be constructed by using more and more accurate, and suitably generalized, Wilson expansions; however, this leads quickly to considerable complications, and is actually an academic exercise as long as even the AF of zeroth degree is not yet really understood.

The AFs, due to their containing unknown functions, do not yet allow any statement on the asymptotic behaviour of the VFs. To make progress in that direction one presently has to resort to assumptions. We discuss in particular the assumptions ${ }^{7}$ made implicitly by Wilson [15] and other authors (for references, see [15]). As there is no apriori (nor, we believe, physical) reason for the correctness of those assumptions, we attempt to construct a consistency argument in their favour. That argument, which uses properties of zero-mass theories, we have not carried through completely. However, because of the importance of the problem, we give the sketch of its present stage.

The first two sections are reviews included for the convenience of the reader. Section I describes the technique of mass vertex insertion. Section II concerns the AFs at nonexceptional momenta and their relation to zero-mass theories, some properties of these theories, and

${ }^{6}$ If one makes full use of the technique described in Appendix B, no asymptotic analysis of any vertex function is required, whereby the most laborious part in the usual renormalization group calculations to determine nonleading logarithms is avoided.

${ }^{7}$ In this context, stronger assumptions are made in QED by Baker and Johnson ([16], and references therein). 
the characterization of exceptional momenta in terms of UR singularities of VFs in zero-mass theories.

In Section III the AFs of some VFs, and of the mass VF, at exceptional momenta are obtained, as well as the AF of second degree of the inverse one-particle propagator. In Section IV we discuss the assumptions, mentioned before, that have been made to arrive at statements on asymptotic behaviour, sketch the attempted consistency check concerning those assumptions, and describe the resulting asymptotic behaviours of VFs. In Appendix A we give an elementary derivation of the WilsonZimmermann formulas [13] used in Section III. In Appendix B properties of the zero-mass theories are discussed in detail, and in particular also the UR singularities relevant for Section III. Appendix C treats the relatively academic problem of mass vertex insertion as non-infinitesimal operation.

\section{Mass Vertex Insertion}

In this paper, for simplicity we only consider the theory of a scalar Hermitean field $\phi$ with Lagrangian density

$$
\begin{aligned}
& L(\phi, \partial \phi)=\frac{1}{2} \partial_{\mu} \phi \partial^{\mu} \phi-\frac{1}{2} m^{2} \phi^{2}-\frac{1}{24} g \phi^{4} \\
& \quad+\frac{1}{2}\left(Z_{3}-1\right)\left(\partial_{\mu} \phi \partial^{\mu} \phi-m^{2} \phi^{2}\right)+\frac{1}{2} Z_{3} \delta m^{2} \phi^{2}-\frac{1}{24}\left(Z_{1}-1\right) g \phi^{4},
\end{aligned}
$$

but all conclusions hold, with small modifications that are then indicated, in QED (cp. Ref. [10]).

The connected Green's functions ${ }^{8}$ are denoted by $G\left(x_{1} \ldots x_{2 n}\right)$ and their amputated one-particle-irreducible parts (VFs) by $\Gamma\left(x_{1} \ldots x_{2 n}\right)$, with $\Gamma(x y)=-G(x y)^{-1}$ in the convolution sense. Their Fourier transforms are introduced by

$$
(2 \pi)^{4} \delta(\Sigma p) \Gamma\left(p_{1} \ldots p_{2 n}\right)=\int d x_{1} \ldots d x_{2 n} e^{i p_{1} x_{1}+\cdots+i p_{2 n} x_{2 n}} \Gamma\left(x_{1} \ldots x_{2 n}\right) .
$$

In renormalizing $[5,19,20]$ the theory (I.1), the renormalization conditions

$$
\begin{gathered}
\left.\Gamma(p(-p))\right|_{p^{2}=m^{2}}=0, \\
{\left.\left[\partial / \partial p^{2}\right] \Gamma(p(-p))\right|_{p^{2}=m^{2}}=i,} \\
\left.\Gamma\left(p_{1} \ldots p_{4}\right)\right|_{\text {symm.pt. }}=-i g
\end{gathered}
$$

are imposed, the symmetry point being defined by $p_{i} p_{j}=\frac{1}{3} m^{2}\left(4 \delta_{i j}-1\right)$.

The one-parameter family of theories with Lagrange function

$$
L^{s}(\phi, \partial \phi)=L(\phi, \partial \phi)-s \frac{1}{2} Z_{3} \Delta m_{u}^{2} \phi^{2}
$$

${ }^{8}$ The concepts and notation used here and later on are described in Refs. [17, 18]. 
leads $[8]$ to VFs

$$
\Gamma^{s}\left(p_{1} \ldots p_{2 n} ; m^{2}, g\right)=Z(s)^{-n} \Gamma\left(p_{1} \ldots p_{2 n} ; m^{2}(s), g(s)\right)
$$

such that

$$
\begin{array}{r}
Z(s)^{n}[\partial / \partial s] \Gamma^{s}\left(p_{1} \ldots p_{2 n} ; m^{2}, g\right) \\
=\left\{\left(d m^{2}(s) / d s\right)\left[\partial / \partial m^{2}(s)\right]+(d g(s) / d s)[\partial / \partial g(s)]-n(d \ln Z(s) / d s)\right\} \\
\cdot \Gamma\left(p_{1} \ldots p_{2 n} ; m^{2}(s), g(s)\right) .
\end{array}
$$

Except in Appendix C, where finite $s$ will be considered, we will need (I.5) only at $s=0$. Using (I.3), we have [8]

$$
\begin{aligned}
& {\left.[\partial / \partial s] \Gamma^{s}\left(p_{1} \ldots p_{2 n}\right)\right|_{s=0} \equiv \Delta \Gamma\left(p_{1} \ldots p_{2 n}\right)} \\
& =-i Z_{3} \Delta m_{u}^{2} \delta_{n 1}-\frac{1}{2} i Z_{3} \Delta m_{u}^{2} \int d k(2 \pi)^{-4} G(k)^{2} \\
& \cdot\left[\Gamma\left(k(-k) p_{1} \ldots p_{2 n}\right)+\text { factorizing terms if } n \geqq 2\right]
\end{aligned}
$$

where $G(k)=-\Gamma(k(-k))^{-1}$. We normalize the inserted mass term such that

$$
\left.\Delta \Gamma(p(-p))\right|_{p^{2}=m^{2}}=-i m^{2}
$$

whereupon all $\Delta \Gamma\left(p_{1} \ldots p_{2 n}\right)$ can be calculated [8]. Setting

where

$$
\Delta \Gamma(00)=-i m^{2} \varphi(g)
$$

$$
\varphi(g)=1+O\left(g^{2}\right)
$$

we show in Appendix A that

$$
\begin{aligned}
& \Delta \Gamma\left(p_{1} \ldots p_{2 n}\right) \\
& \quad=-\frac{1}{2} i m^{2} \varphi(g)\left\langle T N_{2}\left\{\phi(0)^{2}\right\} \tilde{\phi}\left(p_{1}\right) \ldots \tilde{\phi}\left(p_{2 n}\right)\right\rangle^{\text {prop }}
\end{aligned}
$$

in the notation ${ }^{9}$ of Zimmermann [13]. In field operators,

$$
Z_{3} \Delta m_{u}^{2}: \phi(x)^{2}:=m^{2} \varphi(g) N_{2}\left\{\phi(x)^{2}\right\}
$$

i.e. our mass term is a multiple of the finite local operator $N_{2}\left\{\phi(x)^{2}\right\}$.

(I.5) gives ${ }^{10}$ for $s=0$

$$
\begin{aligned}
\Delta & \Gamma\left(p_{1} \ldots p_{2 n} ; m^{2}, g\right) \\
& =\left\{\alpha(g) m^{2}\left[\partial / \partial m^{2}\right]+\beta(g)[\partial / \partial g]-2 n \gamma(g)\right\} \Gamma\left(p_{1} \ldots p_{2 n} ; m^{2}, g\right) \\
& \equiv \mathcal{O} \mu_{2 n} \Gamma\left(p_{1} \ldots p_{2 n} ; m^{2}, g\right)
\end{aligned}
$$

${ }^{9}$ However, our Fourier transforms differ by powers of $2 \pi$ from Zimmermann's.

${ }^{10}$ Arguments for the Eqs. (I.12) were given independently of Ref. [8] by C. G. Callan [21]. 
where

$$
\begin{aligned}
& \alpha(g)=\left.m^{-2}\left(d m^{2}(s) / d s\right)\right|_{s=0}, \\
& \beta(g)=\left.(d g(s) / d s)\right|_{s=0}, \\
& \gamma(g)=\left.\frac{1}{2}(d Z(s) / d s)\right|_{s=0} .
\end{aligned}
$$

These functions are calculated [8] from (I.12) by requiring the conditions (I.2a, b, c) to hold. The result is

$$
\begin{array}{ll}
\alpha(g)=1, & \\
\beta(g)=b_{0} g^{2}+b_{1} g^{3}+\cdots, & b_{0}=3\left(32 \pi^{2}\right)^{-1}, \\
\gamma(g)=c_{0} g^{2}+c_{1} g^{3}+\cdots, & c_{0}=\left(2^{11} 3 \pi^{4}\right)^{-1} .
\end{array}
$$

Occasionally we will write (I.12), using (I.14a), as

$$
\begin{gathered}
\Delta \Gamma\left(p_{1} \ldots p_{2 n} ; m^{2} \lambda^{-2}, g\right) \\
=\left\{-\lambda^{2}\left[\partial / \partial \lambda^{2}\right]+\beta(g)[\partial / \partial g]-2 n \gamma(g)\right\} \Gamma\left(p_{1} \ldots p_{2 n} ; m^{2} \lambda^{-2}, g\right) .
\end{gathered}
$$

\section{Asymptotic Forms, Zero-mass Theory, and Exceptional Momenta}

We wish to study the VFs for large momenta. For the time being, it is convenient to multiply all momenta by a scaling factor $\lambda$ and to consider $\lambda \rightarrow \infty$. For dimensional reasons,

$$
\Gamma\left(\lambda p_{1} \ldots \lambda p_{2 n} ; m^{2}, g\right)=\lambda^{4-2 n} \Gamma\left(p_{1} \ldots p_{2 n} ; m^{2} \lambda^{-2}, g\right)
$$

and likewise for the (dimensionally equal) $\Delta \Gamma$.

A brief discussion of the results of Weinberg [1], Fink [2], and Kinoshita [22] relating to the large- $\lambda$ dependence of (II.1) is given in Ref. [10]. We here immediately proceed to use (I.15). Its inspection suggests to introduce

$$
\varrho(g)=\int_{g_{0}}^{g} d g^{\prime} \beta\left(g^{\prime}\right)^{-1}=-b_{0}^{-1} g^{-1}-b_{0}^{-2} b_{1} \ln g+\varrho_{0}\left(g_{0}\right)+\varrho_{1} g+\cdots .
$$

As (I.14b) must be considered to be a meaningful series (e.g., an asymptotic one) for $g \rightarrow 0$, and since $b_{0}>0, \varrho(g)$ increases from $-\infty$ as $g$ runs from zero through positive values, until it approaches a value $g_{\infty}$ such that ${ }^{11}$ $\lim _{g \rightarrow g_{\infty}} \varrho(g)=\infty$. We will in all of the following, except in Section IV, for

11 The alternative possibility, that $\varrho(g)$ does not increase to $\infty$ for increasing $g$, is briefly commented upon in Section IV.1. 
definiteness choose $g$ to lie inside this interval although we know of no reason why (the renormalized) $g$ should e.g. take positive ${ }^{12}$ rather than negative values. In this interval, the inverse function $\varrho^{-1}$ is unique, and we can define ${ }^{13}$ the monotonically increasing function

$$
g(\lambda)=\varrho^{-1}\left(\ln \lambda^{2}+\varrho(g)\right) .
$$

Its behaviour at $\lambda \rightarrow 0$ is obtained from (II.2) and (II.3) by iterative solution, yielding

$$
\begin{aligned}
g(\lambda)^{-1} & =g^{-1}-b_{0} \ln \lambda^{2}-b_{0}^{-1} b_{1} \ln \left[g^{-1} g(\lambda)\right]+b_{0} \varrho_{1}[g(\lambda)-g]+\cdots \\
& =g^{-1}+b_{0} \ln \lambda^{-2}+b_{0}^{-1} b_{1} \ln \left(1+b_{0} g \ln \lambda^{-2}\right)-b_{0} \varrho_{1} g-\cdots
\end{aligned}
$$

where the omitted terms have a limit for $\lambda \rightarrow 0$. For strictly perturbation theoretical purposes one uses the, otherwise useless, expansion

$$
g(\lambda)=g+\sum_{n=1}^{\infty}(n !)^{-1}\left(\ln \lambda^{2}\right)^{n}[\beta(g) d / d g]^{n-1} \beta(g)
$$

that follows directly from (II.3) and (II.2).

We now define, shortcutting the discussion of (I.15) given in Ref. [10],

$$
\begin{gathered}
\Gamma_{a s}\left(p_{1} \ldots p_{2 n} ; m^{2} \lambda^{-2}, g\right)=\Gamma\left(p_{1} \ldots p_{2 n} ; m^{2} \lambda^{-2}, g\right) \\
-\int_{\lambda^{2}}^{\infty} d \lambda^{\prime 2} \lambda^{\prime-2} \Delta \Gamma\left(p_{1} \ldots p_{2 n} ; m^{2} \lambda^{\prime-2}, g\left(\lambda^{\prime-1} \lambda\right)\right) \\
\quad \cdot \exp \left[2 n \int_{g\left(\lambda^{\prime}-1 \lambda\right)}^{g} d g^{\prime} \beta\left(g^{\prime}\right)^{-1} \gamma\left(g^{\prime}\right)\right]
\end{gathered}
$$

if it exists, which is then easily checked to be a solution of the homogeneous PDE to (I.15), and thus has the form

$$
\Gamma_{a s}\left(p_{1} \ldots p_{2 n} ; m^{2} \lambda^{-2}, g\right)=a(g)^{n} \Phi_{p_{1} \ldots p_{2 n-1}}\left(-\ln m^{2}+\ln \lambda^{2}+\varrho(g)\right)
$$

where

$$
a(g)=\exp \left[2 \int_{0}^{g} d g^{\prime} \beta\left(g^{\prime}\right)^{-1} \gamma\left(g^{\prime}\right)\right]=\exp \left[2 b_{0}^{-1} c_{0} g+\cdots\right] .
$$

Since the last term in (II.6) vanishes for $\lambda \rightarrow \infty$ if it exists at all, we may, in view of (II.1), expect $\lambda^{4-2 n} \Gamma_{a s}\left(p_{1} \ldots p_{2 n} ; m^{2} \lambda^{-2}, g\right)$ to be the asymptotic form (AF) of $\lambda^{4-2 n} \Gamma\left(p_{1} \ldots p_{2 n} ; m^{2} \lambda^{-2}, g\right)$ in the sense of the Introduction, as will be verified below.

We first consider (II.6) in perturbation theory. In all graphs contributing to $\Delta \Gamma$ there is the inserted mass vertex carrying the factor

${ }^{12}$ In QED, the analog of $g$ in this paper is the square of the charge and thus positive. This is the main reason why we do not consider negative $g$ here.

${ }^{13}$ This function is denoted by $g\left(\lambda^{-1}\right)$ in Ref. [10] and should not be confounded with $g(s)$ of (I.4). 
$m^{2} \lambda^{\prime-2}$ due to (I.7). $g\left(\lambda^{\prime-1} \lambda\right)$ involves, due to (II.5), to every finite order in $g$ only finite powers of $\ln \lambda^{\prime}$. Thus, the existence of the $\lambda^{\prime}$-integral is lost only if the momenta are such that the explicit factor $\lambda^{\prime-2}$ from the extra mass vertex is compensated by a quadratic $U R$ divergence stemming from the, in the limit $\lambda^{\prime} \rightarrow \infty$ vanishing, mass $m \lambda^{\prime-1}$. Such (sets of) momenta that the integral does not exist are called exceptional momenta [10] and will be characterized later. At such momenta, the asymptotic exponent $2-2 n$ of $\Delta \Gamma$, in the sense of Weinberg [1], the reduction from $4-2 n$ (cp. (II.1)) being due to the extra propagator denominator, is raised to $4-2 n$, the asymptotically dominant routing [1] of the external momenta through the graph sparing the mass vertex. - Outside of perturbation theory, we note that $g\left(\lambda^{\prime-1} \lambda\right) \rightarrow 0$ as $\lambda^{\prime} \rightarrow \infty$ such that the exponential in (II.6) then has the limit $a(g)^{n}$. While the mass in $\Delta \Gamma$ goes to zero at $\lambda^{\prime} \rightarrow \infty$, the coupling constant does so too, and we shall find below that the rate it does so is just the one consistent with the assumption that it is only at exceptional momenta that $\Delta \Gamma$ in (II.6) is not decreasing like $\lambda^{\prime-2}$ times some power of $\ln \lambda^{\prime}$, or, see Section IV, a power of $\lambda^{\prime}$ smaller than two. This consistency will be more and more apparent as we follow up what it leads to.

Thus, to every finite order in perturbation theory, and more generally in the sense of the foregoing remarks, at nonexceptional momenta

$\Gamma\left(p_{1} \ldots p_{2 n} ; m^{2} \lambda^{-2}, \tilde{g}(\lambda)\right)-\Gamma_{a s}\left(p_{1} \ldots p_{2 n} ; m^{2} \lambda^{-2}, \tilde{g}(\lambda)\right)=O\left(\lambda^{-2}(\ln \lambda)^{\beta}\right)$

where, in perturbation theory, $\tilde{g}(\lambda)$ should not depend on $\lambda$ more strongly than logarithmically ${ }^{14}$, and, outside of perturbation theory, $\tilde{g}(\lambda)$ should not increase as $\lambda \rightarrow \infty$. From (II.9) and (II.7) follows for nonexceptional momenta

$$
\begin{aligned}
& \Gamma_{a s}\left(p_{1} \ldots p_{2 n} ; m^{2}, g\right) \\
& =\lim _{\lambda \rightarrow \infty}\left\{\Gamma\left(p_{1} \ldots p_{2 n} ; m^{2} \lambda^{-2}, g\left(\lambda^{-1}\right)\right) \exp \left[2 n \int_{g\left(\lambda^{-1}\right)}^{g} d g^{\prime} \beta\left(g^{\prime}\right)^{-1} \gamma\left(g^{\prime}\right)\right]\right\} .
\end{aligned}
$$

Thus, $\Gamma_{a s}$ is obtained from $\Gamma$ by taking a zero-mass limit ${ }^{15}$ whereby, however, the coupling constant goes to zero simultaneously and a certain $\lambda$-dependent factor must be applied. This factor has the limit $a(g)^{n}$, however, in perturbation theory, it gives, via (II.5), a power series in $\ln \lambda^{2}$. Since the limit in (II.10) also exists in perturbation theory, where it means that, in each order of $g$, all powers of $\ln \lambda^{2}$ not accompanied by one or more factors $\lambda^{-2}$ cancel, an intricate cancellation of powers of $\ln \lambda^{2}$ takes place. Outside of perturbation theory, (II.10) shows that, for the zero-mass limit to exist, the coupling constant must go to zero

${ }^{14}$ With $\tilde{g}(\lambda)=g$, (II.9) shows that $\Gamma_{a s}$ is the AF of $\Gamma$ in the sense of the Introduction.

15 That the $\Gamma_{a s}$ describe a zero-mass theory can be seen directly from (II.13) below. 
at just the rate at which it goes to zero in $\Delta \Gamma$ in (II.6); in view of the integral equations [8] that define $\Delta \Gamma$ and which contain again essentially only the functions $\Gamma$ (at nonexceptional momenta for them), this is the consistency argument concerning the behaviour of $\Delta \Gamma$ in (II.6) mentioned before.

It is instructive to look at (II.10) in an elementary perturbation theoretical manner. For an irreducible $n \geqq 3$ graph, and momenta nonexceptional in the sense of Weinberg [1], the zero-mass limit exists as the asymptotic exponent is $4-2 n$ and the logarithmic exponent zero, cp. (II.1). When insertions are made in the graph the logarithmic exponent increases [2] due to renormalization momenta being scaled down together with the mass, and the logarithmic terms in (II.10), arising from use of (II.5), are so devised as to cancel all nonzero-exponent terms, with $g(\lambda)$ in the $\Gamma$-argument related to 4-vertex-part insertions and the exponential factor to propagator-corrections insertions. This shows that $\Gamma_{a s}$ is obtainable from $\Gamma$ as follows, referred to later as the "elementary recipe": Replace all momenta $p_{i}$ by $\lambda p_{i}$, factor out $\lambda^{4-2 n}$, and make an expansion, for large $\lambda$, in terms of powers of $\ln \lambda$, powers of $\ln \lambda$ multiplied by $\lambda^{-2}$, by $\lambda^{-4}$, and so on, as in (0.2). The $\lambda$-independent terms in this expansion define $\Gamma_{a s}$, the reason being that $g\left(\lambda^{-1}\right)$ in (II.10) due to (II.5) does not bring negative powers of $\ln \lambda$. On the other hand, $\Gamma_{a s}\left(\lambda p_{1} \ldots \lambda p_{2 n} ; m^{2}, g\right)$ is obtained, by virtue of (II.9), from the described expansion by keeping all terms not containing negative powers of $\lambda$, and is, using (II.7) and (II.1), obtained more simply by

$$
\begin{aligned}
& \Gamma_{a s}\left(\lambda p_{1} \ldots \lambda p_{2 n} ; m^{2}, g\right) \\
& =\lambda^{4-2 n} \Gamma_{a s}\left(p_{1} \ldots p_{2 n} ; m^{2}, g(\lambda)\right) \exp \left[-2 n \int_{g}^{g(\lambda)} d g^{\prime} \beta\left(g^{\prime}\right)^{-1} \gamma\left(g^{\prime}\right)\right] .
\end{aligned}
$$

These recipes are the same ones as the renormalization group method [5] leads to, except that there, $\beta(g)$ and $\gamma(g)$, or $g(\lambda)$ and $a(g)^{-1} a(g(\lambda))$, must be determined by special asymptotic considerations involving the renormalization functions, while here these functions were obtained by a technique, Section I, not involving any hypothesis concerning asymptotic behaviour.

The foregoing recipes show that, in perturbation theory, the introduction of the $\Gamma_{a s}$ is a gain only if they can be obtained directly, avoiding the highly uneconomical "elementary recipe". The obvious direct way is their construction as the VFs of a zero-mass theory, and is described in Appendix B. That in all limit formulas in this section, exceptional momenta had to be avoided (and, in Appendix B, necessitate special provisos) means, see (II.10), that the VFs $\Gamma_{a s}$ of the zero-mass theory are singular at exceptional momenta in virtue of the zeroness of the physical 
mass, while for finite mass, for (II.10) to be meaningful, there should not be a singularity at such momenta.

We first consider Euclidean momenta ${ }^{16}$. Ruelle [23] has shown that VFs are regular ${ }^{17}$ at such momenta if the mass is positive, and so also for zero-mass except where some nontrivial partial sum of momenta vanishes. Thus, Euclidean exceptional momenta are at most those where some such partial sum vanishes. In the model (I.1), inspection of Feynman graphs shows that these partial sums must be even ones for an unboundedness to arise, in virtue of phase space and selection rules. Furthermore, the singularity will be logarithmic if only one such partial sum vanishes; if two nonoverlapping such partial sums vanish the singularity will be quadratic, and so on, the degree of $U R$ singularity corresponding to the increase of asymptotic exponent $2 \alpha$ in $(0.2)$ over $4-2 n$. Since $\Delta \Gamma$, to which, cp. (I.10), Ruelle's analysis also applies, has already one "even" vertex at zero momentum, one vanishing nontrivial even partial sum of momenta leads already to quadratic $U R$ singularity and thus to divergence of the integral in (II.6), in conformity with the nonexistence of $\Gamma_{a s}$ at such momenta due to logarithmic $U R$ singularity. This, as well as the detailed discussion of examples in Section III, points to the consistency of the assumptions concerning behaviour at exceptional momenta, made earlier in this section, also outside perturbation theory.

For real Minkowski momenta, Ruelle [23] has shown that VFs are regular except where the square of a nontrivial partial sum of momenta lies on the relevant mass spectrum. However, at such momenta, the VFs need not actually be singular, e.g., $\Gamma(p(-p))$ is generally regular away from mass thresholds, such that in all finite orders of perturbation theory only lightlike $p$ is an exceptional momentum. More generally, lightlike partial sums of momenta play a role comparable to vanishing partial sums of momenta in the Euclidean case discussed before. Expressed differently, if momenta become large we have, with respect to the $\lambda$-dependence in (0.1), an "essentially Euclidean" situation if no squares of such momenta, or of partial sums of such momenta, do not become (absolutely) large, or if, in coordinate space, approaches to coinciding arguments are from space- or timelike, but not from lightlike directions (cp., e.g. Ref. [13]). At the present time, however, we cannot give a general discussion of Minkowskian exceptional momenta.

The zero-mass theory is discussed in Appendix B. Here we only make two observations [10]. First, all members of the family of zero-mass

16 These are momenta such that the Gram matrix $p_{i} p_{j}$ has real elements and is negative semidefinite and of rank $\leqq 4$. Such momenta can be realized by vectors the zeroth components of which are imaginary, the other components real.

17 We disregard here and below the possible singularities brought about through the amputations. There are none such in perturbation theory. 
theories considered here are related to each other by dilatation and renormalizing the VFs, as follows from (II.11) or also (II.10) and as will be discussed in terms of renormalization conditions in Appendix B. Second, the zero-mass theory is a particle theory ${ }^{18}$ since its VFs can be conventionally normalized. Namely, (II.7) with (II.1) yields

$$
\begin{aligned}
& \left(\lambda^{2} p^{2}\right)^{-1} \Gamma_{a s}\left(\lambda p(-\lambda p) ; m^{2}, g\right) \\
& \quad=\left(p^{2}\right)^{-1} \Gamma_{a s}\left(p(-p) ; m^{2}, g(\lambda)\right) \exp \left[2 \int_{g(\lambda)}^{g} d g^{\prime} \beta\left(g^{\prime}\right)^{-1} \gamma\left(g^{\prime}\right)\right] .
\end{aligned}
$$

Letting $\lambda \rightarrow 0$ gives on the r.h.s. the asymptotic VF of the interaction-free theory, which is $i p^{2}$, such that hereby the 1.h.s. becomes $i a(g)$, showing that the $\phi \ldots$ in (II.7) are, for $\lambda=1$, conventionally normalized zeromass-theory VFs. This theory possesses scattering amplitudes since $p_{i}^{2}=0, \forall i$, although not "essentially Euclidean", appears for general sets of momenta not to be exceptional. - However, in (II.2) we made use of $g(\lambda) \rightarrow 0$ as $\lambda \rightarrow 0$, such that the correct normalization cannot be verified in perturbation theory where (II.5) is relevant, and where (obtainable, e.g., from (II.12) directly in the form (II.14))

$$
\begin{aligned}
& a(g)^{-1} \Gamma_{a s}\left(p(-p) ; m^{2}, g\right) \\
& \quad=i p^{2} \cdot\left[1+\sum_{k=1}^{\infty} \sum_{l=0}^{k-1} a_{k l} g^{k}\left(\ln \left(-p^{2} / m^{2}\right)\right)^{l}\right] .
\end{aligned}
$$

(II.7) shows, however, that the r.h.s. equals

$$
\lambda^{2} a(g(\lambda))^{-1} \Gamma_{a s}\left(\lambda^{-1} p\left(-\lambda^{-1} p\right) ; m^{2}, g(\lambda)\right)
$$

such that, choosing $\lambda^{2}=-m^{-2} p^{2}$ and using (II.13)

$$
a(g)^{-1} \Gamma_{a s}\left(p(-p) ; m^{2}, g\right)=i p^{2}\left\{1+\sum_{k=1}^{\infty} a_{k 0}\left[g\left(\left(-p^{2} / m^{2}\right)^{\frac{1}{2}}\right)\right]^{k}\right\}
$$

which, with (II.5) inserted, is a reordering of (II.13). In (II.14) the summation that makes (II.4) applicable can be performed and so the claimed normalization verified.

To conclude this section, we note that, using (II.1), (II.9), and (II.7)

$$
\begin{aligned}
\lambda^{2 n-4}\left[\Gamma\left(\left(\lambda p_{1}+r_{1}\right) \ldots\left(\lambda p_{2 n}+r_{2 n}\right) ; m^{2}, g\right)-\Gamma\left(\lambda p_{1} \ldots \lambda p_{2 n} ; m^{2}, g\right)\right] \\
=a(g)^{n} a(g(\lambda))^{-n}\left[\Gamma_{a s}\left(\left(p_{1}+\lambda^{-1} r_{1}\right) \ldots\left(p_{2 n}+\lambda^{-1} r_{2 n}\right) ; m^{2}, g(\lambda)\right)\right. \\
\left.\quad-\Gamma_{a s}\left(p_{1} \ldots p_{2 n} ; m^{2}, g(\lambda)\right)\right]+O\left(\lambda^{-2}(\ln \lambda)^{\beta}\right)
\end{aligned}
$$

18 The alternative would have been an "infra particle" theory [24], such as QED with respect to the electrons. In Ref. [10] it is shown that zero-mass QED is "infra" at least with respect to the photons. 
for sufficiently large $\lambda$, with $\Sigma p=\Sigma r=0$, provided the momenta $p_{i}$ are nonexceptional, because the set of sets of such momenta is open. Since the $\Gamma_{a s}$ are regular at such momenta, the square bracket on the r.h.s. is $O\left(\lambda^{-1}\right)$ for $\lambda$ finite. As $\lambda$ increases, $g(\lambda)$ increases and the properties of the $\Gamma_{a s}$ become uncertain, as discussed in Section IV, however, in all finite orders of perturbation theory $g(\lambda)$ gives, due to (II.5), rise only to finite powers of $\ln \lambda$ such that in all orders of perturbation theory the 1.h.s. in (II.15) vanishes as $\lambda \rightarrow \infty$, which affirms the statements made on $(0.1)$ and $(0.2)$ in the Introduction at least in all orders of perturbation theory. In Section III we shall find that those statements are not true for exceptional sets of momenta $p_{i}$.

\section{Asymptotic Forms at Exceptional Momenta}

The reason for the nonexistence of $\Gamma_{a s}$ in (II.6) at exceptional momenta is that then in (I.15) $\Delta \Gamma$ is not negligible relative to $\Gamma$. Thus, we have to analyse $\Delta \Gamma$ asymptotically, since it is only at $\lambda \rightarrow \infty$ that we hope to profit from (I.15). Once its (true) AF $\Delta \Gamma_{\underline{a} \underline{s}}$ (as discussed in Section II, $\Delta \Gamma_{a s}$ never exists) is found and inserted into (I.15) in place of $\Delta \Gamma$, we shall see that the resulting PDE is always easily solved by Ansatz, leading to the (true) AF $\Gamma_{\underline{a s}}$ of $\Gamma$, to be contrasted with the nonexisting $\Gamma_{a s}$ of (II.7) or (II.10). We will discuss in detail only the simplest cases, sufficient to display the technique.

\section{III.1. Four-point Vertex Function}

For $\Gamma\left(\left(\lambda p_{1}+r_{1}\right) \ldots\left(\lambda p_{4}+r_{4}\right)\right)$ with $\Sigma p=\Sigma r=0$, according to Section II the momenta are exceptional if and, to the extent as we consider only a Euclidean situation, only if $p_{1}+p_{2}=p_{3}+p_{4}=0$ etc., i.e. for

$$
\Gamma\left(\left(\lambda p+r_{1}\right)\left(-\lambda p+r_{2}\right)\left(\lambda q+r_{3}\right)\left(-\lambda q+r_{4}\right)\right) .
$$

We first consider $q=0, p \neq 0$ (and $p^{2} \neq 0$ ). (A.11) gives

$$
\begin{aligned}
G(\lambda p & \left.+r_{1}\right) G\left(-\lambda p+r_{2}\right) \Gamma\left(\left(\lambda p+r_{1}\right)\left(-\lambda p+r_{2}\right) r_{3} r_{4}\right) \\
& =\left\langle T N_{2}\left\{\tilde{\phi}\left(\lambda p+r_{1}\right) \tilde{\phi}\left(-\lambda p+r_{2}\right)\right\} \tilde{\phi}\left(r_{3}\right) \tilde{\phi}\left(r_{4}\right)\right\rangle^{\text {prop }} \\
& +G\left(\lambda p+\frac{1}{2}\left(r_{1}-r_{2}\right)\right) G\left(-\lambda p+\frac{1}{2}\left(r_{2}-r_{1}\right)\right) . \\
& \cdot \Gamma\left(\left(\lambda p+\frac{1}{2}\left(r_{1}-r_{2}\right)\right)\left(-\lambda p+\frac{1}{2}\left(r_{2}-r_{1}\right)\right) 00\right) . \\
& \cdot \frac{1}{2}\left\langle T N_{2}\left\{\phi(0)^{2}\right\} \tilde{\phi}\left(r_{3}\right) \tilde{\phi}\left(r_{4}\right)\right\rangle^{\text {prop }} .
\end{aligned}
$$

As $\lambda \rightarrow \infty$ the first term on the r.h.s. behaves like $\lambda^{-6}(\ln \lambda)^{\beta}$ and thus is asymptotically negligible relative to the second term, which like the l.h.s. behaves like $\lambda^{-4}(\ln \lambda)^{\beta^{\prime}}$. Leaving the detailed discussion of the last factor 
in (III.1) (whose nontrivial $r_{3}, r_{4}$ dependence proves the nonvanishing of the 1.h.s. of (II.15) as $\lambda \rightarrow \infty$ for the present exceptional momenta) till Section III.2, we see that we need only consider $\Gamma(p(-p) 00)$ further. (I.12) and (I.10) give

$$
\begin{aligned}
\mathcal{O} p_{4} & \Gamma(p(-p) 00)=\Delta \Gamma(p(-p) 00) \\
= & -\frac{1}{2} i m^{2} \varphi(g)\left\langle T N_{2}\left\{\phi(0)^{2}\right\} \tilde{\phi}(p) \tilde{\phi}(-p) \tilde{\phi}(0) \tilde{\phi}(0)\right\rangle^{\text {prop }} \\
= & -\frac{1}{2} i m^{2} \varphi(g) \Gamma(p(-p) 00) \cdot \\
& \quad \cdot \frac{1}{2} \int d x\left\langle T N_{2}\left\{\phi(x)^{2}\right\} N_{2}\left\{\phi(0)^{2}\right\} \tilde{\phi}(0) \tilde{\phi}(0)\right\rangle^{\text {prop }}+\text { negligible }
\end{aligned}
$$

where (A.12) is used. Since the "negligible" term vanishes for $p \rightarrow \infty$ like $p^{-2}(\ln p)^{\beta},($ III.2) gives the homogeneous PDE

$$
\left[\mathcal{O} p_{4}-\eta(g)\right] \Gamma_{a s}(p(-p) 00)=0
$$

where we have introduced (cp. (I.14b) ${ }^{19}$

$$
\begin{aligned}
\eta(g) & =-\frac{1}{4} i m^{2} \varphi(g) \int d x\left\langle T N_{2}\left\{\phi(x)^{2}\right\} N_{2}\left\{\phi(0)^{2}\right\} \tilde{\phi}(0) \tilde{\phi}(0)\right\rangle^{\text {prop }} \\
& =\frac{1}{3} b_{0} g+O\left(g^{2}\right) .
\end{aligned}
$$

(III.3) is solved by

$$
\Gamma_{\underline{a s}}(p(-p) 00)=a(g)^{2} h(g) \Psi_{p}\left(-\ln m^{2}+\varrho(g)\right)
$$

with $a(g)$ from (II.8) and

$$
h(g)=\exp \left[\int_{g_{1}}^{g} d g^{\prime} \beta\left(g^{\prime}\right)^{-1} \eta\left(g^{\prime}\right)\right]=g^{\frac{1}{3}}(1+O(g))
$$

with $g_{1}$ appropriately chosen. (III.5) entails

$$
\begin{aligned}
& \Gamma_{\underline{a s}}\left(\lambda p(-\lambda p) 00 ; m^{2}, g\right) \\
& \quad=\Gamma_{\underline{a s}}\left(p(-p) 00 ; m^{2}, g(\lambda)\right) \exp \left[-\int_{g}^{g(\lambda)} d g^{\prime} \beta\left(g^{\prime}\right)^{-1}\left(4 \gamma\left(g^{\prime}\right)+\eta\left(g^{\prime}\right)\right)\right]
\end{aligned}
$$

to be contrasted with (II.11), and $\Gamma_{\underline{a}}$ is obtained e.g. from

$$
\begin{aligned}
\Gamma_{\underline{a s}}\left(p(-p) 00 ; m^{2}, g\right) \\
=\lim _{\lambda \rightarrow \infty}\left\{\Gamma\left(p(-p) 00 ; m^{2} \lambda^{-2}, g\left(\lambda^{-1}\right)\right) .\right. \\
\left.\quad \cdot \exp \left[\int_{g\left(\lambda^{-1}\right)}^{g} d g^{\prime} \beta\left(g^{\prime}\right)^{-1}\left(4 \gamma\left(g^{\prime}\right)+\eta\left(g^{\prime}\right)\right)\right]\right\}
\end{aligned}
$$

${ }^{19}$ Note that all parametric functions of $g$ to be introduced in this section are, as those in (I.9) and (I.14), real since all PDEs hold in particular at Euclidean coordinate space points where the VFs and $\Delta \Gamma$ are real. 
to be contrasted with (II.10). Although the "elementary recipe" given after (II.10) is ${ }^{20}$ again applicable, (III.8) shows that the limit in (II.10) does now not exist, but that an extra factor (using (III.5) and (II.4)) $h(g) h\left(g\left(\lambda^{-1}\right)\right)^{-1} \sim h(g)\left(b_{0} \ln \lambda^{2}\right)^{\frac{1}{3}}$ must be inserted to compensate for the singularity of $\Gamma_{a s}$ at the exceptional point where, as we see here, $\Gamma_{a s}$ actually vanishes. The singularity of the zero-mass-theory function will be analysed directly in Appendix B, cp. (B.12).

As to the sum of "leading logarithms", since $\Gamma_{\underline{a s}}=-i g(1+O(g))$, (III.7) gives with (III.6)

$$
\Gamma_{\underline{a s}}\left(\lambda p(-\lambda p) 00 ; m^{2}, g\right)=-i g\left(1-b_{0} g \ln \lambda^{2}\right)^{-\frac{2}{3}}+\text { nonleading }
$$

while for nonexceptional momenta, the exponent is -1 (see [8], Section I.3). The reduction of the exponent can be mnemotechnically described as: Of the three channels, one is exceptional (i.e. transmits zero momentum).

We turn to $p \neq 0,(p \pm q)^{2} \neq 0, p^{2} \neq 0, q^{2} \neq 0$ in $\Gamma\left(\left(\lambda p+r_{1}\right)\left(-\lambda p+r_{2}\right)\right.$ $\left.\left(\lambda q+r_{3}\right)\left(-\lambda q+r_{4}\right)\right)$ where for simplicity we will only consider $r_{1}=r_{2}$ $=r_{3}=r_{4}=0$; the formulas for the more general case give a dependence of the AF besides on $p$ and $q$ only on $r_{1}+r_{2}=-r_{3}-r_{4}$ and are straightforward generalizations (I.12) gives

$$
\begin{aligned}
\mathcal{O} p_{4} & \Gamma(p(-p) q(-q)) \\
\quad & =-\frac{1}{2} i m^{2} \varphi(g)\left\langle T N_{2}\left\{\phi(0)^{2}\right\} \tilde{\phi}(p) \tilde{\phi}(-p) \tilde{\phi}(q) \tilde{\phi}(-q)\right\rangle^{\text {prop }}(\mathrm{III} .10) \\
& =i \kappa(g) \Gamma(p(-p) 00) \Gamma(q(-q) 00)+\text { negligible }
\end{aligned}
$$

with

$$
\begin{aligned}
\kappa(g) & =-\frac{1}{8} m^{2} \varphi(g) \iint d x d y\left\langle T N_{2}\left\{\phi(x)^{2}\right\} N_{2}\left\{\phi(y)^{2}\right\} N_{2}\left\{\phi(0)^{2}\right\}\right\rangle^{\text {prop }} \\
& =\frac{1}{3} b_{0}+O(g)
\end{aligned}
$$

where the last step in (III.10) involves (A.12) applied twice. Into

$$
\mathcal{O} \kappa_{4} \Gamma_{\underline{a s}}(p(-p) q(-q))=i \kappa(g) \Gamma_{\underline{a s}}(p(-p) 00) \Gamma_{\underline{a s}}(q(-q) 00)
$$

from (III.10) we insert (III.5) and obtain ${ }^{21}$ easily

$$
\begin{aligned}
& \Gamma_{\underline{a s}}\left(p(-p) q(-q) ; m^{2}, g\right) \\
& \quad=i a(g)^{-2} h(g)^{-2} k(g) \Gamma_{\underline{a s}}\left(p(-p) 00 ; m^{2}, g\right) \Gamma_{\underline{a s}}\left(q(-q) 00 ; m^{2}, g\right) \\
& \quad+a(g)^{2} \Psi_{p q}\left(-\ln m^{2}+\varphi(g)\right)
\end{aligned}
$$

${ }^{20}$ However, the $\mathrm{AF}$ at exceptional momenta are not obtainable by specializing the $\mathrm{AF}$ at general (i.e., nonexceptional) momenta, as comparison of (III.5) with (II.7) shows, and as was discussed in Section II.

21 The case $p= \pm q$ is presumably solved by (III.13) with $\kappa\left(g^{\prime}\right) \rightarrow 2 \kappa\left(g^{\prime}\right)$ but we have not proven this. 
whereof the first term is a particular solution of the inhomogeneous equation, while the second term is the general solution (cp. (II.7)) of the homogeneous one, and we have introduced

$$
k(g)=\int_{g_{2}}^{g} d g^{\prime} \beta\left(g^{\prime}\right)^{-1} \kappa\left(g^{\prime}\right) a\left(g^{\prime}\right)^{2} h\left(g^{\prime}\right)^{2}=-g^{-\frac{1}{3}}(1+O(g))
$$

with $g_{2}$ appropriately chosen, and use of (III.6), (III.11), and (I.14b). (III.13) gives, written in full,

$$
\begin{aligned}
\Gamma_{\underline{a s}}\left(p(-p) q(-q) ; m^{2}, g\right) \\
=\lim _{\lambda \rightarrow \infty} \exp \left[4 \int_{g\left(\lambda^{-1}\right)}^{g} d g^{\prime} \beta\left(g^{\prime}\right)^{-1} \gamma\left(g^{\prime}\right)\right]\left\{\Gamma\left(p(-p) q(-q) ; m^{2} \lambda^{-2}, g\left(\lambda^{-1}\right)\right)\right. \\
\quad+i \int_{g\left(\lambda^{-1}\right)}^{g} d g^{\prime} \beta\left(g^{\prime}\right)^{-1} \kappa\left(g^{\prime}\right) \exp \left[\int_{g\left(\lambda^{-1}\right)}^{g^{\prime}} d g^{\prime \prime} \beta\left(g^{\prime \prime}\right)^{-1}\left(4 \gamma\left(g^{\prime \prime}\right)+2 \eta\left(g^{\prime \prime}\right)\right)\right] . \\
\left.\left.\quad \cdot \Gamma\left(p(-p) 00 ; m^{2} \lambda^{-2}, g\left(\lambda^{-1}\right)\right) \Gamma\left(q(-q) 00 ; m^{2} \lambda^{-2}, g\left(\lambda^{-1}\right)\right)\right\} . \quad \text { III. } 15\right)
\end{aligned}
$$

This shows, comparing with (II.10), that the singularity of $\Gamma_{a s}$ must be removed by a subtraction, rather than compensated by a factor as in (III.8), but that the "elementary recipe" remains valid since the subtractive term does not contribute thereby. The factor of $\Gamma \Gamma$ in the curly bracket goes for $\lambda \rightarrow \infty$ like $i g\left(\lambda^{-1}\right)^{-1}$, thus giving, using (III.8), as subtraction a term $g\left(\lambda^{-1}\right)^{-\frac{1}{3}}$ times a product that has a limit by itself, such that $\Gamma_{a s}$ is infinite at the singularity $(p(-p) q(-q)), p \neq q \neq 0$ rather than zero as at $(p(-p) 00)$ as we found from (III.8). The extraction of the singular part of the zero-mass-theory function will be performed in Appendix B, cp. (B.14).

\section{III.2. Mass Vertex}

We call mass vertex the last factor in (III.1) and write

$$
\frac{1}{2}\left\langle T N_{2}\left\{\phi(0)^{2}\right\} \tilde{\phi}\left(r_{3}\right) \tilde{\phi}\left(r_{4}\right)\right\rangle^{\text {prop }}=\hat{\Gamma}\left(r_{3} r_{4} ; m^{2}, g\right)
$$

For this function, $\left(r_{3}+r_{4}\right)^{2} \neq 0$ characterizes nonexceptional momenta. For any momenta, we have

$$
\begin{gathered}
\mathcal{O} \mu_{0} \hat{\Gamma}\left(r_{3} r_{4}\right)=-\frac{1}{4} i m^{2} \varphi(g) \int d x\left\langle T N_{2}\left\{\phi(x)^{2}\right\} N_{2}\left\{\phi(0)^{2}\right\} \tilde{\phi}\left(r_{3}\right) \tilde{\phi}\left(r_{4}\right)\right\rangle^{\text {prop }} \\
+\frac{1}{4} i m^{2} \varphi(g) \hat{\Gamma}\left(r_{3} r_{4}\right) \int d x\left\langle T N_{2}\left\{\phi(x)^{2}\right\} N_{2}\left\{\phi(0)^{2}\right\} \tilde{\phi}(0) \tilde{\phi}(0)\right\rangle^{\text {prop }} \text {. (III.17) }
\end{gathered}
$$

This is derived ${ }^{22}$ by writing the $N_{2}\left\{\phi(0)^{2}\right\}$ part of (III.16) as a cut-off momentum space integral with cut-off dependent factor, and applying

${ }^{22}$ Another derivation of (III.17) results from applying $\mathcal{O} \mu_{4}$ to both sides of (III.1), with $r_{1}=r_{2}=O$, and equating the terms on both sides that do not vanish for $\lambda \rightarrow \infty$. 
(I.12). Hereby also the ( $m$ - and $g$-dependent) factor must be differentiated, which gives rise to a multiple of $\hat{\Gamma}$, the coefficient being obtained from the normalization condition [13] $\hat{\Gamma}(00)=1$ and occurring already in (III.4).

Inspection of graphs shows that the first integral in (III.17) is negligible relative to the homogeneous term for $r_{3} \rightarrow \infty, r_{4} \rightarrow \infty$, unless these momenta are exceptional, i.e. $\left(r_{3}+r_{4}\right)^{2} \rightarrow \infty$. Thus, in the nonexceptional case

leading to

$$
\left[\mathcal{O} p_{0}+\eta(g)\right] \hat{\Gamma}_{a s}\left(r_{3} r_{4} ; m^{2}, g\right)=0
$$

$$
\hat{\Gamma}_{a s}\left(r_{3} r_{4} ; m^{2}, g\right)=h(g)^{-1} \hat{\Psi}_{r_{3} r_{4}}\left(-\ln m^{2}+\varrho(g)\right)
$$

and

$$
\begin{aligned}
& \hat{\Gamma}_{a s}\left(r_{3} r_{4} ; m^{2}, g\right) \\
& \quad=\lim _{\lambda \rightarrow \infty}\left\{\hat{\Gamma}\left(r_{3} r_{4} ; m^{2} \lambda^{-2}, g\left(\lambda^{-1}\right)\right) \exp \left[-\int_{g\left(\lambda^{-1}\right)}^{g} d g^{\prime} \beta\left(g^{\prime}\right)^{-1} \eta\left(g^{\prime}\right)\right]\right\}
\end{aligned}
$$

with again the "elementary recipe" being applicable.

In the exceptional case, we only consider $r_{3}=\lambda q+s_{3}, r_{4}=-\lambda q+s_{4}$, $q^{2} \neq 0$, and will moreover set $s_{3}=s_{4}=0$ for simplicity. Then, using (A.12), (III.17) reduces to

$$
\left[\mathcal{O} \mu_{0}+\eta(g)\right] \hat{\Gamma}_{\underline{a s}}\left(q(-q) ; m^{2}, g\right)=i \kappa(g) \Gamma_{\underline{a s}}\left(q(-q) 00 ; m^{2}, g\right)
$$

where we have used (III.11). Using (III.3) we obtain easily

$$
\begin{aligned}
& \hat{\Gamma}_{\underline{a s}}\left(q(-q) ; m^{2}, g\right) \\
& \quad=i a(g)^{-2} h(g)^{-2} k(g) \Gamma_{\underline{a s}}\left(q(-q) 00 ; m^{2}, g\right) \\
& \quad+h(g)^{-1} \Theta_{q}\left(-\ln m^{2}+\varrho(g)\right)
\end{aligned}
$$

where (III.14) is used and the last term is the general solution of the homogeneous PDE. The relation to the zero-mass theory is, written in detail,

$$
\begin{array}{r}
\hat{\Gamma}_{\underline{a s}}\left(q(-q) ; m^{2}, g\right) \\
=\lim _{\lambda \rightarrow \infty} \exp \left[-\int_{g\left(\lambda^{-1}\right)}^{g} d g^{\prime} \beta\left(g^{\prime}\right)^{-1} \eta\left(g^{\prime}\right)\right]\left\{\hat{\Gamma}\left(q(-q) ; m^{2} \lambda^{-2}, g\left(\lambda^{-1}\right)\right)(\mathrm{III}\right. \\
+i \int_{g\left(\lambda^{-1}\right)}^{g} d g^{\prime} \beta\left(g^{\prime}\right)^{-1} \kappa\left(g^{\prime}\right) \exp \left[\int_{g\left(\hat{\lambda}^{-1}\right)}^{g^{\prime}} d g^{\prime \prime} \beta\left(g^{\prime \prime}\right)^{-1}\left(4 \gamma\left(g^{\prime \prime}\right)+2 \eta\left(g^{\prime \prime}\right)\right)\right] . \\
\left.\cdot \Gamma\left(q(-q) 00 ; m^{2} \lambda^{-2}, g\left(\lambda^{-1}\right)\right)\right\}
\end{array}
$$


which shows that $\hat{\Gamma}_{a s}$ defined through use of (III.18), does not exist at the exceptional point, its singularity being removed in (III.21) by a subtraction similar as in (III.15), though the "elementary recipe" remains valid. The factor of $\Gamma$ in (III.21) being the same as of $\Gamma \Gamma$ in (III.15), the subtractive term in the curly bracket in (III.21) is, using (III.8), proportional to $g\left(\lambda^{-1}\right)^{-\frac{2}{3}}$ as $\lambda \rightarrow \infty$, or, including the overall factor, proportional to $g\left(\lambda^{-1}\right)^{-1} \sim b_{0} \ln \lambda^{2}$. The extraction of that singularity in the zero-mass theory will be shown in Appendix B, cp. (B.16).

We insert (III.20) into (III.1) and obtain for $\left|p^{2}\right| \gg\left|q^{2}\right| \gg m^{2}$

$$
\begin{aligned}
\Gamma_{\underline{a s}}\left(p(-p) q(-q) ; m^{2}, g\right) & \\
\quad & i a(g)^{-2} h(g)^{-2} k(g) \Gamma_{\underline{a s}}\left(p(-p) 00 ; m^{2}, g\right) \Gamma_{\underline{a s}}\left(q(-q) 00 ; m^{2}, g\right) \\
& +h(g)^{-1} \Gamma_{\underline{a s}}\left(p(-p) 00 ; m^{2}, g\right) \Theta_{q}\left(-\ln m^{2}+\varrho(g)\right) .
\end{aligned}
$$

While the first term on the r.h.s. is the same as in (III.13), the second one differs from the one in (III.13), inspite of (III.5), as a consequence of the unsymmetric asymptotics ${ }^{23}$. Namely, while $\Psi_{p q}$ is symmetric in $p$ and $q$, (III.22) is not, since due to $\Gamma_{\underline{a s}}=-i g(1+O(g))$ and $\hat{\Gamma}_{\underline{a s}}=1+O\left(g^{2}\right)$ from (III.22), (III.6), and (III.11) follows

$$
\Theta_{q}\left(-\ln m^{2}+\varrho(g)\right)=2 g^{\frac{1}{3}}(1+O(g))
$$

which differs from

$$
\Psi_{q}\left(-\ln m^{2}+\varrho(g)\right)=-i g^{\frac{2}{3}}(1+O(g))
$$

obtained from (III.5) with (III.6). Reinserting (III.23) into (III.20), with $m^{2} \rightarrow m^{2} \lambda^{-2}$ gives with (III.9)

$\hat{\Gamma}\left(\lambda q(-\lambda q) ; m^{2}, g\right)$ - nonleading terms

$$
\begin{aligned}
& =i g^{-\frac{2}{3}} \frac{1}{3}\left(-3 g^{-\frac{1}{3}}\right)(-i g)\left(1-b_{0} g \ln \lambda^{2}\right)^{-\frac{2}{3}}+2 g^{-\frac{1}{3}} g^{\frac{1}{3}}\left(1-b_{0} g \ln \lambda^{2}\right)^{-\frac{1}{3}} \\
& =-\left(1-b_{0} g \ln \lambda^{2}\right)^{-\frac{2}{3}}+2\left(1-b_{0} g \ln \lambda^{2}\right)^{-\frac{1}{3}}=1-6 c_{0} g^{2}\left(\ln \lambda^{2}\right)^{2}+\cdots(
\end{aligned}
$$

(cp. (I.14)) which can to this order easily be checked directly.

\section{III.3. One-particle Propagator}

From (I.10) and (III.16) we have

$$
\mathcal{O} p_{2} \Gamma(p(-p))=\Delta \Gamma(p(-p))=-i m^{2} \varphi(g) \hat{\Gamma}(p(-p)) .
$$

Denoting the AF of second degree of $\Gamma(p(-p))$, which includes the terms $O(1)$, as $\Gamma_{\underline{\underline{a s}}}(p(-p))$, we have from (III.25)

$$
\mathcal{O} \kappa_{2} \Gamma_{\underline{a s}}(p(-p))=-i m^{2} \varphi(g) \hat{\Gamma}_{\underline{a s}}(p(-p)) .
$$

${ }^{23}$ In Weinberg's [1] terminology, we are now treating $\Gamma\left(\eta_{1} \eta_{2} p\left(-\eta_{1} \eta_{2} p\right) \eta_{2} q\left(-\eta_{2} q\right)\right)$, rather than $\Gamma(\eta p(-\eta p) \eta q(-\eta q))$ in (III.14). 
Inserting here (III.20) with (III.5) we find easily by Ansatz

$$
\begin{aligned}
& \Gamma_{\underline{\underline{a s}}}\left(p(-p) ; m^{2}, g\right)=\Gamma_{a s}\left(p(-p) ; m^{2}, g\right) \\
& -i m^{2} a(g) h(g) e^{-\varrho(g)} \int_{0}^{g} d g^{\prime} \beta\left(g^{\prime}\right)^{-1} \varphi\left(g^{\prime}\right) e^{\varrho\left(g^{\prime}\right)} a\left(g^{\prime}\right)^{-1} h\left(g^{\prime}\right)^{-1} \cdot \quad(\text { III. } 27) \\
& \cdot\left[\hat{\Gamma}_{\underline{a s}}\left(p(-p) ; m^{2}, g\right)+i a(g)^{-2} h(g)^{-2}\left(k\left(g^{\prime}\right)-k(g)\right) \Gamma_{\underline{a s}}\left(p(-p) 00 ; m^{2}, g\right)\right] \\
& +a(g) \Xi_{p}\left(-\ln m^{2}+\varrho(g)\right)
\end{aligned}
$$

where the last term is the general solution of the homogeneous PDE and must be set zero, in virtue of our choice of integration constants, as we will now prove ${ }^{24}$.

For any $c(g)$ that is $C^{\infty}$ for $g>0$ and bounded at $g \rightarrow 0$ by a negative power we have by repeated partial integration, using (II.6),

$$
e^{-\varrho(g)} \int_{0}^{g} d g^{\prime} \beta\left(g^{\prime}\right)^{-1} e^{\varrho\left(g^{\prime}\right)} c\left(g^{\prime}\right)=\sum_{n=0}^{\infty}[-\beta(g) d / d g]^{n} c(g)
$$

as an asymptotic series. Using this in (III.27) shows that the first two terms on the r.h.s. possess power series expansions in $g$ and reduce, as $g \rightarrow 0$, to the correct value $i p^{2}-i m^{2}$ of the 1.h.s. Thus, the last term on the r.h.s. must have a power series expansion in $g$ and vanish for $g \rightarrow 0$, and must for $m^{2} \rightarrow 0$ vanish in all orders of $g$ since then the asymptotic behaviour of the 1.h.s. is correctly given already by the first term on the r.h.s. alone, in virtue of its definition, the other terms giving only $m^{2}\left[\ln \left(p^{2} / m^{2}\right)\right]^{\beta}$ corrections. Because of (II.8), $\Xi_{p}\left(-\ln m^{2}+\varrho(g)\right)$ must be a power series in $g\left(\left(-p^{2} / m^{2}\right)^{\frac{1}{2}}\right)=g+b_{0} g^{2} \ln \left(-p^{2} / m^{2}\right)+\cdots$ with only $p^{2}$-dependent coefficients. The vanishing at $m^{2} \rightarrow 0$ then allows to conclude the vanishing of the coefficients one by one, q.e.d.

\section{III.4. Other Vertex Functions}

From (A.11) we get easily

$$
\begin{aligned}
& \Gamma\left(\left(\lambda p+r_{1}\right)\left(-\lambda p+r_{2}\right) r_{3} \ldots r_{2 n}\right) \\
& \quad=\Gamma(\lambda p(-\lambda p) 00) \frac{1}{2}\left\langle T N_{2}\left\{\phi(0)^{2}\right\} \tilde{\phi}\left(r_{3}\right) \ldots \tilde{\phi}\left(r_{2 n}\right)\right\rangle^{\text {prop }}+O\left(\lambda^{-1}(\ln \lambda)^{\beta}\right)
\end{aligned}
$$

for $\lambda \rightarrow \infty, p^{2} \neq 0$, the main term going like $(\ln \lambda)^{\beta^{\prime}}$ as we showed in Section III.1. (III.29) shows to which extent (II.15) is violated and, if $n \geqq 3$, the asymptotic behaviour $\lambda^{4-2 n}(\ln \lambda)^{\beta^{\prime \prime}}$ in the case of nonexceptional momenta $p_{i}$ surpassed, as a consequence of the $U R$ - divergence of order $2 n-4$ of the zero-mass-theory function $\Gamma_{a s}(p(-p) 0 \ldots 0)$.

${ }^{24}$ It can be proved even simpler by inserting in (II.6) the $\mathrm{AF}$ of $\Delta \Gamma$, whereupon the last term in (III.27) does not appear. The way over (III.26) is, however, more instructive. 
If one wishes in the present case to obtain the $\mathrm{AF}$ of second degree, i.e. to order $\lambda^{-2}$, not only must in (III.29) the last term also be analysed, for which it suffices to use Zimmermann's [13] $\delta=4$ formulas, instead of $\delta=2$ in (III.29), but the AFs of $\Gamma(\lambda p(-\lambda p) 00)$ and of the now appearing additional functions

and

$$
\begin{gathered}
\Gamma(\lambda p(-\lambda p) 0000), \\
\left.\left(\partial / \partial r_{\mu}\right) \Gamma\left(\left(\lambda p-\frac{1}{2} r\right)\left(-\lambda p-\frac{1}{2} r\right) r 0\right)\right|_{r=0}, \\
\left.\left(\partial^{2} / \partial r_{\mu} \partial r_{v}\right) \Gamma\left(\left(\lambda p-\frac{1}{2} r\right)\left(-\lambda p-\frac{1}{2} r\right) r 0\right)\right|_{r=0},
\end{gathered}
$$

$$
\left.\left(\partial^{2} / \partial r_{\mu} \partial s_{v}\right) \Gamma\left(\left(\lambda p-\frac{1}{2}(r+s)\right)\left(-\lambda p-\frac{1}{2}(r+s)\right) r s\right)\right|_{r=s=0}
$$

to accuracy $\lambda^{-2}$ are needed, which are, for all these functions, the AFs of second degree. To obtain these AFs, one again uses mass vertex insertion and expands the $\Delta \Gamma$ according to $\delta=4$ formulas [13]. One thereby obtains a coupled system of PDEs which we will, however, not discuss here.

It is obvious that, if one wishes to obtain the AF of a VF where e.g. two pairs of arguments become large at the same rate (cp. (III.13)) or at different rates (cp. (III.22)), suitable generalizations and, possibly, extensions of the technique of Sections III.1 and III.2 should be used. Ultimately, one might thereby arrive at such a detailed description of the asymptotic behaviour, in terms of AFs that do suffice in all finite orders of perturbation theory, with logarithmic terms completely described, as envisaged by Weinberg [1], and this in principle, cp. (III.27), for not only the lowest degree.

\section{Considerations on Asymptotic Behaviour}

The AFs obtained in Sections II and III do not yet yield statements on the asymptotic behaviour of the functions in question because we obtained no information on the functions $\phi_{p_{1}}\left(-\ln m^{2}+\ln \lambda^{2}+\varrho(g)\right)$ of (II.7) and corresponding ones in Sections III.1 and III.2, except that these are VFs and mass vertex functions and, in the case of exceptional momenta, their nonsingular parts, of a certain zero-mass theory, as discussed in Appendix B. That information does not relate, however, to the $\lambda \rightarrow \infty$ behaviour of those functions.

One method available now is perturbation theory. It yields easily the leading logarithmic, next to leading logarithmic, etc., approximations of these functions, samples of which are (III.9), (III.24), and formulas in Section I.3 of Ref. [8], and obviously of no use for $\lambda \rightarrow \infty$. Since no other rational technique than perturbation theory is yet available, one may resort to assumptions. 


\section{IV.1. Preliminary Discussion}

We combine (II.11), (II.1) and (II.9) to

$$
\begin{aligned}
\Gamma\left(\lambda p_{1} \ldots \lambda p_{2 n} ; m^{2}, g\right) \\
=\lambda^{4-2 n} \Gamma_{a s}\left(p_{1} \ldots p_{2 n} ; m^{2}, g(\lambda)\right) \exp \left[-2 n \int_{g}^{g(\lambda)} d g^{\prime} \beta\left(g^{\prime}\right)^{-1} \gamma\left(g^{\prime}\right)\right] \\
\quad+O\left(\lambda^{2-2 n}(\ln \lambda)^{\beta}\right)
\end{aligned}
$$

valid (concerning the last term, in all finite orders of perturbation theory) for nonexceptional momenta. (IV.1) is applicable for arbitrarily large $\lambda$ only if $g(\lambda)$ is defined, i.e. if the integral in (II.2) diverges for $g \rightarrow g_{\infty}$ from below, where $g_{\infty}$ is the first ${ }^{25}$ zero of $\beta(g)$ for positive $g$, or $g_{\infty}=\infty$ for positive $\beta(g)$. This is violated if for $\beta(g)$ e.g. the approximation by the first term in (I.14b) is inserted. No inconsistency of the theory would follow yet, but (II.11) would define an extension of $\Gamma_{a s}$ (which should, in virtue of (II.6), exist for all $g$ for which $\Gamma$ exists) in $g$ into regions where it was not originally defined or, if defined, assumed in the original definition different values. An inconsistency [25] can only be construed if assumptions on $\Gamma_{a s}$ are made that in effect exclude such behaviour. Such assumptions are suggested by the interpretation of $\Gamma_{a s}$ as zeromass-theory VF. Therefore, we only consider the more comfortable situation with $\varrho(g) \rightarrow \infty$ for $g \rightarrow g_{\infty}$ further.

The possibility that $g_{\infty}$ is finite ${ }^{26}$ was noted by Gell-Mann and Low [4]. The integral in the exponent in (IV.1) is, using (II.2) and (II.3),

$$
\int_{g}^{g(\lambda)} d g^{\prime} \beta\left(g^{\prime}\right)^{-1} \gamma\left(g^{\prime}\right)=\gamma\left(g_{\infty}\right) \ln \lambda^{2}+\int_{g}^{g(\lambda)} d g^{\prime} \beta\left(g^{\prime}\right)^{-1}\left[\gamma\left(g^{\prime}\right)-\gamma\left(g_{\infty}\right)\right]
$$

provided $\gamma\left(g_{\infty}\right)$ exists. The last integral may or may not converge as $\lambda \rightarrow \infty$ but it will in any case be negligible relative to the first term on the r.h.s., which gives in (IV.1) a factor $\lambda^{-4 n \gamma\left(g_{\infty}\right)}$. This factor governs the asymptotic behaviour in (IV.1) if $\Gamma_{a s}\left(p_{1} \ldots p_{2 n} ; m^{2}, \mathrm{~g}_{\infty}\right)$ exists, and this is now assumed (implicitly in Ref. [15], and together with more restrictive assumptions in QED ${ }^{27}$ in Ref. [16]) in view of the perturbation series in $g_{\infty}$ of that function.

The hook is that the last argument is apriori unfounded. Namely, also the exponential factor in (IV.1) is for $\lambda \rightarrow \infty$ a power series in $g_{\infty}$ (times a power series in $g$ ) and only what we know more about it reveals

${ }^{25}$ We forego, for brevity, the discussion of more general possibilities. Cp., e.g., Ref. [15].

${ }^{26}$ All of the following holds also if $g_{\infty}=+\infty$, however, then the assumptions necessary to make further look less natural.

${ }^{27}$ In QED (see Ref. [8]), the analogous assumption for the inverse photon propagator, together with assumed finiteness of $e_{\infty}^{2}$, implies the finiteness of the bare charge (cp. Refs. $[4,16])$. The bare coupling constant in the present model is discussed in Section IV.4. 
that under the assumptions mentioned before it diverges. Extracting from $\Gamma_{a s}\left(p_{1} \ldots p_{2 n} ; m^{2}, g(\lambda)\right)$ a factor $[f(g(\lambda))]^{n}$ where $f(g(\lambda))$ is a power series in $g(\lambda)$ of peculiar behaviour as $g(\lambda) \rightarrow g_{\infty}$, and postulating for the remainder a $g(\lambda) \rightarrow g_{\infty}$ limit would mean to declare zero-mass-theory functions normalized differently than those given by $\Gamma_{a s}$ to be more appropriate for a description of that theory in the $g \rightarrow g_{\infty}$-limit.

For the present model, we showed in Section II that the VFs

$$
a(g(\lambda))^{-n} \Gamma_{a s}\left(p_{1} \ldots p_{2 n} ; m^{2}, g(\lambda)\right)=\Phi_{p_{1} \ldots p_{2 n-1}}\left(-\ln m^{2}+\ln \lambda^{2}+\varrho(g)\right)
$$

are conventionally normalized VFs of the zero-mass theory such that, forming these in (IV.1) and assuming for them a $g(\lambda) \rightarrow g_{\infty}$ limit (which, of course, does not exist in perturbation theory with respect to $g$ ), the phenomenon of changed power behaviour as $\lambda \rightarrow \infty$ disappears. In view of

$\Phi_{p_{1} \ldots p_{2 n-1}}\left(-\ln m^{2}+\ln \lambda^{2}+\varrho(g)\right)=a(g)^{-n} \Gamma_{a s}\left(p_{1} \ldots p_{2 n} ; m^{2} \lambda^{-2}, g\right)$

however, the limit functions would necessarily be scale invariant, and Pohlmeyer [26] has shown that scale-invariant zero-mass theories are free ones if they satisfy certain axiomatic requirements. Since we see no reason to abandon these requirements, in (IV.1) the factor of $\lambda^{4-2 n}$ would thus vanish for $\lambda \rightarrow \infty$, if $n \geqq 2$, making the assumption discussed here, which would lead to "canonical dimension" of the field operator ${ }^{28}$ in the present usage of that term (see, e.g. [27]), complicated to pursue further. In this context, it should be noted that the possibility of conventional normalization of the $\Gamma_{a s}$ is special to the present model and does not exist e.g. in QED [10], and thus appears incidental and not connected with large-momenta behaviour.

While this remark only discredits a finite $\lambda \rightarrow \infty$ limit of (IV.3), there is arbitrariness in the assumption that $\Gamma_{a s}\left(p_{1} \ldots p_{2 n} ; m^{2}, g_{\infty}\right)$ exists. In this situation, one may resort to (II.6), which we write

$$
\begin{aligned}
& \Gamma_{a s}\left(p_{1} \ldots p_{2 n} ; m^{2}, g(\lambda)\right)=\Gamma\left(p_{1} \ldots p_{2 n} ; m^{2}, g(\lambda)\right) \\
& \quad-\int_{1}^{\infty} d \mu^{2} \mu^{-2} \Delta \Gamma\left(p_{1} \ldots p_{2 n} ; m^{2} \mu^{-2}, g\left(\lambda \mu^{-1}\right)\right) \exp \left[2 n \int_{g\left(\lambda \mu^{-1}\right)}^{g(\lambda)} d g^{\prime} \beta\left(g^{\prime}\right)^{-1} \gamma\left(g^{\prime}\right)\right] .
\end{aligned}
$$

As $\lambda \rightarrow \infty$ we may suppose the first term on the right hand side to have a limit since for the nonasymptotic theory, $g_{\infty}$ is not expected to be a peculiar value of the coupling constant, although it is one for the asymptotic theory since, to obtain such zero-mass theory from the family of zero-mass theories discussed in Appendix B requires an infinite dilata-

${ }^{28}$ For present interpretations of, e.g., deep-inelastic electron-proton scattering "canonical dimensions" need apply only in certain cases of exceptional momenta without necessarily requiring the limiting zero-mass theory discussed above to exist. 
tion, see, e.g. (II.11). Thus, the interest centers on the integral in (IV.4), which becomes for $\lambda \rightarrow \infty$

$$
\int_{1}^{\infty} d \mu^{2} \mu^{-2-4 n \gamma\left(g_{\infty}\right)} \Delta \Gamma\left(p_{1} \ldots p_{2 n} ; m^{2} \mu^{-2}, g_{\infty}\right)
$$

and again one may assume the integrand to exist as the definition of $\Delta \Gamma$ through integral equations (see Ref. [8]) does not involve the asymptotic theory, however, the convergence of the integral is now in question. It is comforting that there is now some latitude in admissable asymptotic behaviour of $\Delta \Gamma\left(\ldots ; m^{2} \mu^{-2}, g_{\infty}\right)$ for $\mu \rightarrow \infty$, i.e., rather weaker statements on the asymptotic behaviour of $\Delta \Gamma$ suffice to support stronger statements on the asymptotic behaviour of $\Gamma\left(\ldots ; m^{2} \lambda^{-2}, g\right)$ as $\lambda \rightarrow \infty$ but we are dealing with a different function the asymptotic behaviour of which must be analysed separately. Clearly, this will require new assumptions, and so forth.

\section{IV.2. Consistency Argument}

We will show here that under certain conditions, it is consistent to assume that certain asymptotic functions have limits as $g \rightarrow g_{\infty}$. We consider only the inverse propagator since the necessary calculations were performed in Section III.3. If we specialize (IV.4) to that case, we see that the integral, with $\Delta \Gamma$ replaced by its $A F$, was already evaluated in (III.27) with the last term to be omitted and $g$ to be replaced by $g(\lambda)$, the left hand side being the closer approximation to $\Gamma$ than $\Gamma_{a s}$.

We must now assume that not only $\gamma\left(g_{\infty}\right)$ but also $\eta\left(g_{\infty}\right)$ exists. Then, assuming for simplicity that the last integral in (IV.2) converges and also the corresponding one with $\gamma(g)$ replaced by $\eta(g)$, one proves by direct estimate under suitable continuity assumptions that

$$
\begin{array}{r}
\lim _{g \rightarrow g_{\infty}} a(g) h(g) e^{-\varrho(g)} \int_{0}^{g} d g^{\prime} \beta\left(g^{\prime}\right)^{-1} \varphi\left(g^{\prime}\right) e^{\varrho\left(g^{\prime}\right)} a\left(g^{\prime}\right)^{-1} h\left(g^{\prime}\right)^{-1} \\
=\left[1-2 \gamma\left(g_{\infty}\right)-\eta\left(g_{\infty}\right)\right]^{-1} \varphi\left(g_{\infty}\right)
\end{array}
$$

if that exists and the denominator is positive. Likewise one obtains

$$
\begin{aligned}
& \lim _{g \rightarrow g_{\infty}} a(g)^{-1} h(g)^{-1} e^{-\varrho(g)} \\
& \cdot \int_{0}^{g} d g^{\prime} \beta\left(g^{\prime}\right)^{-1} \varphi\left(g^{\prime}\right) e^{\varrho\left(g^{\prime}\right)} a\left(g^{\prime}\right)^{-1} h\left(g^{\prime}\right)^{-1} \int_{g}^{g^{\prime}} d g^{\prime \prime} \beta\left(g^{\prime \prime}\right)^{-1} \kappa\left(g^{\prime \prime}\right) a\left(g^{\prime \prime}\right)^{2} h\left(g^{\prime \prime}\right)^{2} \\
& \quad=-\left[1-\left(2 \gamma\left(g_{\infty}\right)+\eta\left(g_{\infty}\right)\right)^{2}\right]^{-1} \kappa\left(g_{\infty}\right) \varphi\left(g_{\infty}\right)
\end{aligned}
$$


if that exists and the denominator is positive. Thus, assuming

$$
\Gamma_{a s}\left(p(-p) ; m^{2}, g\right), \quad \Gamma_{\underline{a s}}\left(p(-p) 00 ; m^{2}, g\right),
$$

and $\hat{\Gamma}_{a s}\left(p(-p) ; m^{2}, g\right)$ all to have limits as $g \rightarrow g_{\infty}$ is, under the conditions just given and presupposing $\Gamma\left(p(-p) 00 ; \mathrm{m}^{2}, g\right)$ to exist thereby, consistent on the present level. Of course, a sharpening of this consistency test would require increasingly many asymptotic and simply parametric functions to have limits as $g \rightarrow g_{\infty}$. In the following, we abstract from the special case considered here.

\section{IV.3. Statement and Discussion of Assumptions}

The consequences of the following assumptions for asymptotic behaviour will be given in Section IV.4:

Assumption $A$ : All functions $\Gamma$ and mass vertex functions $\hat{\Gamma}$ in the finitemass theory have limits for $g \rightarrow g_{\infty}$ at fixed momenta. (Remark: the reason for including mass vertex functions will be discussed below. $g_{\infty}$ is defined by

$$
\int^{g_{\infty}} d g^{\prime} \beta\left(g^{\prime}\right)^{-1}=+\infty, \quad g_{\infty} \neq 0 .
$$

Assumption B: All functions of $g$ alone that are directly obtained from perturbation expansions, rather than in solving a PDE of the type (I.12), have limits for $g \rightarrow g_{\infty}$. Furthermore, the limiting values of some of these functions obey certain constraints. (Remark: Examples are $\beta(g)$, with $\beta\left(g_{\infty}\right)=0$ if $g_{\infty}<\infty, \gamma(g), \varphi(g), \eta(g), \kappa(g)$, against $\varrho(g), a(g)$, $h(g), k(g)$, the constraints being of the variety needed for (IV.5).)

Assumption $C$ : The following functions have limits for $g \rightarrow g_{\infty}$ at fixed momenta

a) all asymptotic functions $\Gamma_{a s}$ and certain mass vertex functions $\hat{\Gamma}_{a s}$ at nonexceptional momenta

b) all asymptotic functions $\Gamma_{\underline{a}}$ and certain mass vertex functions $\hat{\Gamma}_{a s}$ at exceptional momenta at which the corresponding $\Gamma_{a s}$ and $\hat{\Gamma}_{a s}$ are logarithmically singular. (Remark: We may define those functions that were not defined precisely in Section III, by the "elementary recipe" since it was found to yield the correct result in those cases but cannot be expected to be valid at stronger than logarithmic singularities of the $\Gamma_{a s}$ or $\hat{\Gamma}_{a s}$. This is the reason for the restriction made, rather than the expectation that the assumption concerning the limit would be unsound for more singular cases.)

Assumptions A and B are natural ones as $g_{\infty}$ is apriori no peculiar value of the coupling constant. Clearly, B is more comfortable if $g_{\infty}$ 
is finite [4]. Assumption C, however, is much too strong to be directly acceptable. Therefore, we replace it by

Assumption $C^{\prime}$ : a) $\Gamma_{a s}\left(p(-p) ; m^{2}, g\right)$ and $\Gamma_{a s}\left(p_{1} \ldots p_{4} ; m^{2}, g\right)$ have at $p^{2}=-m^{2}$ and at the symmetry point $p_{i} p_{j}=-\frac{1}{3}\left(4 \delta_{i j}-1\right) m^{2}$ respectively, limits as $g \rightarrow g_{\infty}$.

b) Certain functions of $g$ alone, defined by limiting processes from the finite-mass theory, have limits as $g \rightarrow g_{\infty}$.

From $\mathrm{C}^{\prime}$ a), we can consider all functions $\Gamma_{a s}$ for arbitrary momenta being constructed in the way described in Appendix B for the conditions (B.1), in a perturbation expansion if nothing better is available, in terms of the new renormalization conditions of the zero-mass theory described by the $\Gamma_{a s}$. Formulas $(\mathrm{B} .12,14,16,17)$ then allow to define the $\Gamma_{a s}, \hat{\Gamma}_{a s}$, and $\hat{\Gamma}_{\underline{a}}$ directly in terms of that theory provided the functions $v(g)$, and $\chi\left(r, s ; m_{2}, g\right), \sigma\left(r^{2} m^{-2}, g\right), \tau\left(r^{2} m^{-2}, g\right)$, the latter e.g. for $r^{2}=-m^{2}$ and $s=r$, have been obtained and have the property stated in $\mathrm{C}^{\prime} \mathrm{b}$ ). It would be desirable to obtain these functions directly for $g=g_{\infty}$ in terms of functions of the limiting theory and so to replace assumption $C^{\prime} b$ ) by a weaker one. To this end, one would in formulas analogous to $(\mathrm{B} .4,13,15)$ set both total momenta occuring nonzero and would go to the limit of one total momentum zero through a limiting process within the zeromass theory. With this, and (B.16), in mind we included also mass vertex functions in Assumption C.a).

The result of the consideration in Section IV.2 and of the foregoing observations is that the Assumptions $\mathrm{A}, \mathrm{B}$, and $\mathrm{C}$, or preferably $\mathrm{C}^{\prime}$, are consistent.

The $g \rightarrow g_{\infty}$ limiting theory has the property that follows from (II.11) and (IV.2) ${ }^{29}$

$\Gamma_{a s}\left(\mu p_{1} \ldots \mu p_{2 n} ; m^{2}, g_{\infty}\right)=\mu^{4-2 n-4 n \gamma\left(g_{\infty}\right)} \Gamma_{a s}\left(p_{1} \ldots p_{2 n} ; m^{2}, g_{\infty}\right)$

such that it is scale-invariant ${ }^{30}$ with, however, "change of dimension" unless $\gamma\left(\mathrm{g}_{\infty}\right)=0$, a case that would imply the asymptotic theory to be the free one [26] met already in Section IV.1. Clearly, the theory admits conventional normalization only in this special case. From (III.7) follows

$\Gamma_{\underline{a s}}\left(\mu p(-\mu p) 00 ; m^{2}, g_{\infty}\right)=\mu^{-8 \gamma\left(g_{\infty}\right)-2 \eta\left(g_{\infty}\right)} \Gamma_{\underline{a s}}\left(p(-p) 00 ; m^{2}, g_{\infty}\right)$

which conforms with (IV.6), using (B.9-11), for a zero-mass theory. Similar simple transformation formulas hold for the other $\Gamma_{\underline{a s}}$ and for $\hat{\Gamma}_{a s}$ and $\hat{\Gamma}_{\underline{a s}}$.

${ }^{29}$ It is not necessary to assume that the last integral in (IV.2) converges as $g(\lambda) \rightarrow g_{\infty}$.

${ }^{30}$ It appears that a local zero-mass theory that satisfies (IV.6) should also be conformal-invariant with the same anomalous dimension $1+2 \gamma\left(g_{\infty}\right)$. This was suggested to the author by $\mathrm{K}$. Wilson, to whom the author is indebted for a discussion of this point. 
While the present $\Gamma_{a s}$ and $\Gamma_{a s}$ have, e.g. using the "elementary recipe", expansions in $g_{\infty}$, (IV.6) and (IV.7) do not, of course, hold in those expansions, as they would otherwise be identities in $g_{\infty}$ with the exponents some power series in $g_{\infty}$, and consistency with (II.11), which is an identity for the series representing $\Gamma_{a s}$, would require the exponent to be $g_{\infty}$ independent and thus to be precisely $4-2 n$. That $\Gamma_{a s}$ is not, however, scale invariant identically in $g$ is trivial, cp. (II.13). Thus, with the $\Gamma_{a s}$ in (IV.6) defined ${ }^{31}$, in principle, by the expansions in $g_{\infty}$ (IV.6) represents a condition on $g_{\infty}$ from which, one could think, $g_{\infty}$ together with the exponent could be determined without need of referring back to $\beta(g)$ and $\gamma(g)$. Such use of (IV.6) might be related to fixed-point concepts of Wilson [15].

\section{IV.4. Possible Types of Asymptotic Behaviour}

Under the assumptions of Section IV.3, (IV.1) gives with (IV.2) for nonexceptional momenta ${ }^{32}$

$$
\begin{aligned}
\Gamma\left(\lambda p_{1}\right. & \left.\ldots \lambda p_{2 n} ; m^{2}, g\right) \\
& =\lambda^{4-2 n-4 n \gamma\left(g_{\infty}\right)} r(g)^{-2 n} \omega(\lambda)^{-2 n} \Gamma_{a s}\left(p_{1} \ldots p_{2 n} ; m^{2}, g_{\infty}\right)+O\left(\lambda^{2-2 n}(\ln \lambda)^{\beta}\right)
\end{aligned}
$$

where the $r(g)$ - and $\omega(\lambda)$-factors come from the last term in (IV.2), and $\omega(\lambda)$ will, if not constant for $\lambda \rightarrow \infty$, not be as strongly $\lambda$-dependent as a power. For exceptional momenta, e.g., $\Gamma\left(\lambda p(-\lambda p) 00 ; m^{2}, g\right)$ a similar formula deducible from (III.7) holds. From positive-definiteness [28] in the case $n=1$ follows that $\gamma\left(g_{\infty}\right) \geqq 0$. For $\gamma\left(g_{\infty}\right)=0$, the first term on the r.h.s. of (IV.8) is zero for $n \geqq 2$, and proportional to $p^{2}$ for $n=1$, because of (IV.6). Among the possibilities here under consideration, this one appears us to be the most attractive. It corresponds to a vanishing bare coupling constant (for the definition, see Ref. [10], Section VI), while $\gamma\left(g_{\infty}\right)>0$ would lead to an ambiguous bare coupling constant.

As an example of a possibly non-power-type asymptotic behaviour, we consider the mass vertex of Section III.2; the result for

$$
\Gamma\left(\lambda p(-\lambda p) \lambda q(-\lambda q) ; m^{2}, g\right),
$$

31 To use instead the more direct definition of the zero-mass theory given in Appendix B, (IV.7) should, with the help of (B.2), be transscribed into a relation involving $\Gamma_{0}$ and $V_{\infty}=V\left(g_{\infty}\right)$.

32 (IV.8) shows that if in (II.10) on the r.h.s. $g\left(\lambda^{-1}\right)$ is replaced by $g$ and $g$ by $g(\lambda)$, that side would, under the assumptions validating (IV.8), have a limit (though not in perturbation expansion in $g$ ) with on the l.h.s. $g$ replaced by $g_{\infty}$. - In (IV.8), the last term on the r.h.s. can, for $n=1$, not be inferred from (III.27) and could also be larger than indicated, since it is open how fast $\Gamma_{a s}\left(p_{1} \ldots p_{2 n} ; m^{2}, g(\lambda)\right)$ should approach its supposed limiting value as $g(\lambda) \rightarrow g_{\infty}$, which is a peculiar value of this parameter in the zero-mass theory. 
on the basis of (III.13), cp. (III.15), would be similar. From the consequence of (III.20)

$$
\begin{array}{r}
\hat{\Gamma}_{\underline{a s}}\left(\lambda q(-\lambda q) ; m^{2}, g\right)=h(g)^{-1} h(g(\lambda)) \hat{\Gamma}_{\underline{a s}}\left(q(-q) ; m^{2}, g(\lambda)\right) \\
-i(k(g(\lambda))-k(g)) a(g(\lambda))^{-2} h(g(\lambda))^{-1} h(g)^{-1} \Gamma_{\underline{a s}}\left(q(-q) 00 ; m^{2}, g(\lambda)\right)
\end{array}
$$

follows: If $2 \gamma\left(g_{\infty}\right)+\eta\left(g_{\infty}\right)>0$, the asymptotic behaviour is

$$
\begin{aligned}
& \lambda^{2 \eta\left(g_{\infty}\right)} \exp \left[\int_{g}^{g(\lambda)} d g^{\prime} \beta\left(g^{\prime}\right)^{-1}\left(\eta\left(g^{\prime}\right)-\eta\left(g_{\infty}\right)\right)\right] \\
& \cdot\left\{\hat{\Gamma}_{\underline{a} s}\left(q(-q) ; m^{2}, g_{\infty}\right)-\frac{1}{2}\left(2 \gamma\left(g_{\infty}\right)+\eta\left(g_{\infty}\right)\right)^{-1} \kappa\left(g_{\infty}\right) \Gamma_{\underline{a s}}\left(q(-q) 00 ; m^{2}, g_{\infty}\right)\right\} .
\end{aligned}
$$

If $2 \gamma\left(g_{\infty}\right)+\eta\left(g_{\infty}\right)=0$ the curly bracket herein becomes replaced by $\left\{\hat{\Gamma}_{\underline{a} \underline{s}}\left(q(-q) ; m^{2}, g_{\infty}\right)-i \kappa\left(g_{\infty}\right) \ln \lambda^{2} \Gamma_{\underline{a s}}\left(q(-q) 00 ; m^{2}, g_{\infty}\right)\right\}$.

If $2 \gamma\left(g_{\infty}\right)+\eta\left(g_{\infty}\right)<0$, the dominating term is

$$
\begin{array}{r}
-i h(g)^{-1} \lambda^{-4 \gamma\left(g_{\infty}\right)-2 \eta\left(g_{\infty}\right)} \exp \left[-\int_{g}^{g(\lambda)} d g^{\prime} \beta\left(g^{\prime}\right)^{-1}\left(\theta\left(g^{\prime}\right)-\theta\left(g_{\infty}\right)\right)\right] \quad \text { (IV.10c) } \\
\cdot \int_{g}^{g_{\infty}} d g^{\prime \prime} \beta\left(g^{\prime \prime}\right)^{-1} a\left(g^{\prime \prime}\right)^{2} h\left(g^{\prime \prime}\right)^{2} \kappa\left(g^{\prime \prime}\right) \cdot \Gamma_{\underline{a s}}\left(q(-q) 00 ; m^{2}, g_{\infty}\right)
\end{array}
$$

where

$$
\theta\left(g^{\prime}\right)=4 \gamma\left(g^{\prime}\right)+2 \eta\left(g^{\prime}\right) .
$$

For non-overall scaling, appropriate formulas follow from (II.15), and less trivial ones from (III.1), (III.29), and, more interestingly, (III.22), cp. the remarks made there. It is seen that, although the assumptions of Section IV.3 lead essentially to power-behaviours, the "dimensions" [15] hereby differ in general from the one in the simplest case (IV.8), and, under circumstances as in (IV.10b), non-pure-power behaviours result. The exponents and characteristic constants are independent of the actual coupling constant $g$, which, e.g., in (IV.8) enters the leading term only through the factor $r(g)^{-2 n}$. However, the closer $g$ is to $g_{\infty}$, the sooner will the asymptotic form be reached, because of the $g(\lambda)$-dependence of $\Gamma_{a s}$ in (IV.1).

\section{Conclusion}

After a review of the mass-vertex insertion technique and of previous results on asymptotic forms at nonexceptional momenta and the zeromass theory relating to them, we derived the asymptotic forms of some simple vertex functions and of the mass vertex at exceptional momenta, at which the asymptotic forms for general momenta are inapplicable due to $U R$-singularities of the zero-mass theory. We showed that these new asymptotic forms are related to zero-mass vertex functions from 
which the $U R$-singularities are extracted sometimes in a subtractive, and sometimes in a multiplicative manner.

All these asymptotic forms yield no information on the asymptotic behaviour of the functions considered without knowledge of the behaviour of the zero-mass theory in a certain limit, expressible as an infinite dilatation in a family of zero-mass theories all related to each other by finite dilatations. Concerning this, we discussed some assumptions, and in particular one set of assumptions (which, in QED, would imply the finiteness of the bare charge) for which we gave a consistency argument, with the help of considerations on the zero-mass theory. The resulting asymptotic behaviours are power laws or slight modifications thereof, with the exponents being coupling-constant independent, but these forms are reached the sooner the closer the coupling constant is to a value distinguished in the zero-mass theory. We do not, however, consider the aforementioned particular assumptions as physically compelling.

\section{Appendix A}

\section{Elementary Derivation of Some Expansion Formulas}

We first derive the formula

$$
\begin{array}{r}
\left.\left\langle T: \phi(x) \phi(y): \phi\left(z_{1}\right) \ldots \phi\left(z_{2 n}\right)\right\rangle=\left\langle T N_{2}\{\phi(x) \phi(y)\} \phi\left(z_{1}\right) \ldots \phi\left(z_{2 n}\right)\right\rangle \quad \text { (A. } 1\right) \\
\quad+\langle T: \varphi(x) \phi(y): \tilde{\phi}(0) \tilde{\phi}(0)\rangle^{\text {elem }} \frac{1}{2}\left\langle T N_{2}\left\{\phi\left(\frac{1}{2}(x+y)\right)^{2}\right\} \phi\left(z_{1}\right) \ldots \phi\left(z_{2 n}\right)\right\rangle
\end{array}
$$

in the notation of Zimmermann [13] ${ }^{33}$ extensively used in Section III.

We have $[20,29,30]$ for connected Green's functions

$$
\begin{aligned}
& G\left(x y z_{1} \ldots z_{2 n}\right)=G^{2 i}\left(x y, z_{1} \ldots z_{2 n}\right) \\
& \quad+\iint d u d v G(x y \underline{u} \underline{v})\left[\frac{1}{2} G^{2 i}\left(u v, z_{1} \ldots z_{2 n}\right)\right. \\
& \left.\quad+\sum_{\substack{\text { partitions } \\
k+l=n+1}} G\left(u z_{i_{1}} \ldots z_{i_{2 k-1}}\right) G\left(v z_{j_{1}} \ldots z_{j_{2 l-1}}\right)\right] .
\end{aligned}
$$

Here underlining an argument means propagator amputation, and $G^{2 i}$ is that part of the connected Green's function that is two-particle irreducible between the first and second sets of arguments, with $G^{2 i}\left(x y, z_{1} z_{2}\right) \equiv 0$.

We now use a matrix notation [31] that allows easy manipulations. The Bethe-Salpeter (BS) equation is written

$$
I=B+B G I=B+I G B .
$$

${ }^{33}$ However, we use the Fourier transforms

$$
\tilde{\phi}(p)=\int d x e^{i p x} \phi(x) \text { etc. }
$$


Here / is the 4-point VF, $B$ the BS kernel (which in the model(I.1) involves a logarithmically divergent constant, so that for finiteness we should first consider the regularized theory, see, e.g., Ref. [18], and go to the limit in the final equations), and $G$ stands for the pair of propagators. Momenta are indicated as

$$
\begin{gathered}
\left.\Gamma\left(\left(-\frac{1}{2} q-p\right)\left(-\frac{1}{2} q+p\right)\left(\frac{1}{2} q+p^{\prime}\right)\left(\frac{1}{2} q-p^{\prime}\right)\right) \Leftrightarrow \Leftrightarrow^{p^{q} p^{\prime}}\right) \\
G\left(-\frac{1}{2} q-p\right) G\left(-\frac{1}{2} q+p\right) \Leftrightarrow \stackrel{q}{G}
\end{gathered}
$$

etc., and are suppressed if internal and integrated over (with a factor $\frac{1}{2}(2 \pi)^{-4}$ supplied), or if external (left entry, right exit, and throughgoing or total) and kept general. In (A.3) it is understood that internal momenta fit unless one momentum is indicated to be kept fixed, such that that factor is constant in the internal-momentum integration, as below in (A.7).

Now

$$
G I=\stackrel{0}{G} \stackrel{0}{I}+(G-\stackrel{0}{G}) \stackrel{0}{I}+\stackrel{0}{G}(I-\stackrel{0}{I})
$$

and, using (A.3) twice,

$$
\begin{aligned}
I-\stackrel{0}{I} & =B-\stackrel{0}{B}+(I-\stackrel{0}{l}) G B+\stackrel{0}{l}(G-\stackrel{0}{G}) B+\stackrel{0}{i} G(B-\stackrel{0}{B}) \\
& =(1+\stackrel{0}{I})(B-\stackrel{0}{B})(1+G I)+\stackrel{0}{I}(G-\stackrel{0}{G}) I .
\end{aligned}
$$

Together,

$$
1+G I=(1+\stackrel{0}{G} \stackrel{0}{I})[1+\stackrel{0}{G}(B-\stackrel{0}{B})(1+G /)+(G-\stackrel{0}{G})] .
$$

Furthermore, from (A.3)

$$
\stackrel{0}{I}-\stackrel{0}{I^{0}}=\stackrel{0}{B}-\stackrel{0}{B^{0}}+\stackrel{0}{i} \stackrel{0}{G}\left(\stackrel{0}{B}-\stackrel{0}{B^{0}}\right)
$$

such that

$$
\stackrel{0}{I}=\left(\stackrel{0}{I}^{0}+\stackrel{0}{B}-\stackrel{0}{B^{0}}\right)\left[1-\stackrel{0}{G}\left(\stackrel{0}{B}-\stackrel{0}{B}^{0}\right)\right]^{-1}
$$

where the inverse is defined by expansion, and

$$
1+\stackrel{0}{G} \stackrel{0}{I}=\left(1+\stackrel{0}{G}^{0} I^{0}\right)\left[1-\stackrel{0}{G}\left(\stackrel{0}{B}-\stackrel{0}{B}^{0}\right)\right]^{-1} .
$$

Inserting (A.8) into (A.6), we obtain

where

$$
1+G I=\left(1+\stackrel{0}{G}^{0} I^{0}\right)(1+W)
$$

$W=\left[1-\stackrel{0}{G}\left(\stackrel{0}{B}-\stackrel{0}{B^{0}}\right)\right]^{-1}[1+\stackrel{0}{G}(B-\stackrel{0}{B})(1+G /)+(G-\stackrel{0}{G}) /]-1$.

Herefrom

$$
G I=W+\stackrel{0}{G}^{0} I^{0}(1+W)
$$


inserted as $G(x y \underline{u} \underline{v})$ into (A.2) gives (A.1) upon addition of disconnected parts. The kernel $W$ is finite in perturbation theory and allows its left arguments to coincide as power counting [32] easily shows, whereas $G /$ is logarithmically singular thereby. Thus, in (A.10) that singularity is separated off and seen to be factorisable. The formula

$$
\begin{aligned}
& \left\langle T: \phi(x) \phi(y): \tilde{\phi}\left(k_{1}\right) \ldots \tilde{\phi}\left(k_{2 n}\right)\right\rangle^{\text {prop }}=\left\langle T N_{2}\{\phi(x) \phi(y)\} \tilde{\phi}\left(k_{1}\right) \ldots \tilde{\phi}\left(k_{2 n}\right)\right\rangle^{\text {prop }} \\
& \quad+\langle T: \phi(x) \phi(y): \tilde{\phi}(0) \tilde{\phi}(0)\rangle^{\text {elem } \frac{1}{2}\left\langle T N_{2}\left\{\phi\left(\frac{1}{2}(x+y)\right)^{2}\right\} \tilde{\phi}\left(k_{1}\right) \ldots \tilde{\phi}\left(k_{2 n}\right)\right\rangle^{\text {prop }}}
\end{aligned}
$$

in Zimmermann's [13] notation is obtained from (A.1) by removing one-particle reducible parts, and amputation. In (A.11), the first two "prop" can also be replaced by two "elem".

In Section III we use special cases $(n+l=2)$ of

$$
\begin{aligned}
\langle T: \phi(x) & \left.\phi(y): N_{2}\left\{\phi\left(u_{1}\right)^{2}\right\} \ldots N_{2}\left\{\phi\left(u_{l}\right)^{2}\right\} \phi\left(z_{1}\right) \ldots \phi\left(z_{2 n}\right)\right\rangle^{\text {elem }} \\
& =\left\langle T N_{2}\{\phi(x) \phi(y)\} N_{2}\left\{\phi\left(u_{1}\right)^{2}\right\} \ldots N_{2}\left\{\phi\left(u_{l}\right)^{2}\right\} \phi\left(z_{1}\right) \ldots \phi\left(z_{2 n}\right)\right\rangle^{\text {elem }} \\
& +\langle T: \phi(x) \phi(y): \tilde{\phi}(0) \tilde{\phi}(0)\rangle^{\text {elem }} \\
& \cdot \frac{1}{2}\left\langle T N_{2}\left\{\phi\left(\frac{1}{2}(x+y)\right)^{2}\right\} N_{2}\left\{\phi\left(u_{1}\right)^{2}\right\} \ldots N_{2}\left\{\phi\left(u_{l}\right)^{2}\right\} \phi\left(z_{1}\right) \ldots \phi\left(z_{2 n}\right)\right\rangle^{\text {prop }}
\end{aligned}
$$

The definitions, in the approach of Zimmermann [13], of the functions involving several $N_{2}$-operators are for $n+l \geqq 2$ straightforward generalizations of those in (A.1) involving only one $N_{2}$-operator since no new vertex parts arise (in contrast to $l=1, n=0$ ). The definitions of these functions in the spirit of this Appendix require tools developped elsewhere (Section V.2 of Ref. [18]) and are left to the interested reader. In either approach the proof that the first term on the r.h.s. of (A.12) allows $x \rightarrow y$ (unless $l=1, n=0$, for that case, cp. e.g., (III.20)) rests on power counting in precisely the same way as for $l=0$, the only difference being that the vertices on which external momenta act are now, besides three-leg ones, also two-leg ones.

In Section III. $4 \delta>2$ is mentioned. The $\delta=3$-forms ${ }^{34}$ of the $\delta=2$ forms (A.1) and (A.12) can be simply derived as above, whereby now at some steps of the manipulations not only the first but also the second term in a Taylor expansion at zero momentum is subtracted. Only for $\delta \geqq 4$ the present method loses its simplicity due to combinatorial complexities of the variety of, but more cumbersome than, those in Ref. [20]. This also applies to the higher- $\delta$ forms of (A.12).

${ }^{34} \delta$ is the dimension [9] ascribed to the operator in the $N_{\delta}$-product, which characterizes the number of subtractions made when building up the renormalized Feynman integral [13]. When $\delta$ exceeds the actual dimension $d$, subtractions are made that would not be necessary to give a finite Feynman integral. 
We finally prove (I.10). $\Delta \Gamma$ in (I.6) can for $n=1$ be written ${ }^{35}$

$$
\Delta \Gamma=\gamma+\gamma \stackrel{0}{G} \stackrel{0}{i}
$$

where $\gamma=-\mathrm{i} Z_{3} \Delta m^{2}$, the coefficient of the bare vertex, which is taken at zero total momentum. (A.9) gives

$$
\Delta \Gamma=\gamma\left(1+\stackrel{0}{G} \stackrel{0}{ }^{0}\right)\left[1-\stackrel{0}{G}\left(\stackrel{0}{B}-\stackrel{0}{B^{0}}\right)\right]^{-1}=\Delta \Gamma^{0}(1+\stackrel{0}{W})
$$

which is, using (I.8), (I.10) for $n=1$. (I.10) for $n>1$ then follows by use of (A.2).

\section{Appendix B}

\section{Properties of the Zero-Mass Theory}

We noted in Section II that it is economic, and found in Section IV.2 that it is significant, to be able to deal directly with the zero-mass theory as such. This construction will now be described.

For the VFs $\Gamma_{0}$ of the zero-mass theory we choose the renormalization conditions ${ }^{36}$

$$
\begin{gathered}
\Gamma_{0}\left(00 ; U^{2}, V\right)=0, \\
\left.\Gamma_{0}\left(p(-p) ; U^{2}, V\right)\right|_{p^{2}=-U^{2}}=-i U^{2}, \\
\left.\Gamma_{0}\left(p_{1} p_{2} p_{3} p_{4} ; U^{2}, V\right)\right|_{\text {symm.p. } .}=-i V
\end{gathered}
$$

where $U^{2}$ is a mass squared, $V$ dimensionless, and the symmetry point is defined by $p_{i} p_{j}=-\frac{1}{3}\left(4 \delta_{i j}-1\right) U^{2}$. The recursive construction [33] of the VFs $\Gamma_{0}\left(p_{1} \ldots p_{2 n} ; U^{2}, V\right)$ as power series in $V$ proceeds for $n \geqq 3$ in the usual way. For $n=1$ and $n=2$, the final subtraction must be made such as to impose (B.1). We denote the unrenormalized $n=1 \mathrm{VF}$, i.e., the one before the final subtraction, by $\Gamma_{u}(p)$ and form the finite function

$$
\begin{aligned}
\Gamma\left(p, p_{0}\right) & =\Gamma_{u}(p)-\left(p-p_{0}\right)_{\mu}\left(\partial / \partial p_{0 \mu}\right) \Gamma_{u}\left(p_{0}\right) \\
& -\frac{1}{2}\left(p-p_{0}\right)_{\mu}\left(p-p_{0}\right)_{v}\left(\partial^{2} / \partial p_{0 \mu} \partial p_{0 v}\right) \Gamma_{u}\left(p_{0}\right)
\end{aligned}
$$

where $\mathrm{p}_{0}^{2}=-U^{2}$, e.g. using

$$
\begin{aligned}
& \Gamma\left(p, p_{0}\right)=\frac{1}{2}\left(p-p_{0}\right)_{\mu}\left(p-p_{0}\right)_{v}\left(p-p_{0}\right)_{\kappa} \cdot \\
& \left.\quad \cdot \int_{0}^{1} d x(1-x)^{2}\left(\partial^{3} / \partial q_{\mu} \partial q_{\nu} \partial q_{\kappa}\right) \Gamma_{u}(q)\right|_{q=p_{0}+x\left(p-p_{0}\right)}
\end{aligned}
$$

${ }^{35}$ Again, for rigour, regularization should be applied in the first stages.

${ }^{36}$ As the possibility of conventional normalization, discussed in Section II, is not verifyable in perturbation theory, we do not attempt to implement it at this point. 
and for the third derivative the expression given by Johnson [20]. In

$$
\Gamma_{0}\left(p(-p) ; U^{2}, V\right) \equiv \Gamma\left(p^{2}\right)=\Gamma\left(p, p_{0}\right)-A\left(p_{0}\right)-p_{\mu} B^{\mu}\left(p_{0}\right)-\frac{1}{2} p_{\mu} p_{v} C^{\mu v}\left(p_{0}\right)
$$

the functions $A, B$, and $C$ are determined from (B.1 a, b), and one obtains

$$
\Gamma\left(p^{2}\right)=\Gamma\left(p, p_{0}\right)-\Gamma\left(0, p_{0}\right)-p_{\mu} B^{\mu}\left(p_{0}\right)+p^{2} \Gamma^{\prime}\left(p_{0}{ }^{2}\right)+2\left(p p_{0}\right)^{2} \Gamma^{\prime \prime}\left(p_{0}{ }^{2}\right)
$$

where

$$
\begin{gathered}
B^{\mu}\left(p_{0}\right)=\left.\left(\partial / \partial p_{\mu}\right) \Gamma\left(p, p_{0}\right)\right|_{p=0}, \\
\Gamma^{\prime}\left(p_{0}{ }^{2}\right)=\left(p_{0}{ }^{2}\right)^{-1}\left[\Gamma\left(p_{0}{ }^{2}\right)+\Gamma\left(0, p_{0}\right)+\frac{1}{2} p_{0 \mu} B^{\mu}\left(p_{0}\right)\right], \\
\Gamma^{\prime \prime}\left(p_{0}{ }^{2}\right)=\frac{1}{4}\left(p_{0}{ }^{2}\right)^{-2} p_{0 \mu} B^{\mu}\left(p_{0}\right),
\end{gathered}
$$

and

$$
\Gamma\left(p_{0}{ }^{2}\right)=\Gamma\left(-U^{2}\right)=-i U^{2} .
$$

For $n=2$ we use

$$
\Gamma_{0}\left(p_{1} \ldots p_{4} ; U^{2}, V\right)=\Gamma_{u}\left(p_{1} \ldots p_{4}\right)-\left.\Gamma_{u}\left(p_{1} \ldots p_{4}\right)\right|_{\text {symm.pt. }}-i V
$$

where $\Gamma_{u}$ is the function before the final subtraction, and the $\Gamma_{u}$-difference is finite and easily calculated in a variety of ways. In these calculations, $\Gamma\left(0, p_{0}\right)$ and $B^{\mu}\left(p_{0}\right)$ are finite, the (according to (II.14), fictitious) $U R$ singularity appearing only in the second derivative at $p=0$ for phase space reasons (cp. (II.13)), and the $p_{0}$-dependence cancels out.

We now discuss the family of theories so constructed. For unchanged theory, $U^{2}$ in (B.1 b) is not fixed since its change can be compensated by a change of normalization of all VFs without change of the physical content of the theory. Then $V$ will change, however, such that simultaneous change of $V$ and $U^{2}$ describable by $V\left(U^{2}\right)$ does not change the theory physically. On the other hand, a change of $U^{2}$ without a change of $V$ means a dilatation and yields a physically different theory. Thus, there is a one-parameter family of physically distinct theories, all related to each other by dilatation, with no intrinsic dimensionless parameter definable. Since such a result is also immediately deduced from the property (II.7) of the 1.h.s. of (II.10), we expect, and will verify below, that the zero-mass theories described by the $\Gamma_{a s}$ can be related to the ones described by the $\Gamma_{0}$. - These conclusions are not altered if the possibility of conventional normalization is exploited: Imposing, e.g., instead of (B.1 b) $\lim _{p^{2} \rightarrow 0}\left(p^{2}\right)^{-1} \Gamma_{0}\left(p(-p) ; U^{2}, V\right)=i$ again makes, for unaltered theory, $V$ a function of $U^{2}$, and as there is no distinguished value for $U^{2}, U^{2}=0$ leading to $V=0$ in (B.1 c) according to Section II, or to $U R$-divergent $V$ in perturbation theory, the impossibility of introducing an intrinsic dimensionless parameter remains. - It must be noted that the conclusions of this paragraph depend on the non-scale-invariance of the zero-mass 
theory (a scale invariant one anyway not being of interest [26]) which holds in perturbation theory, see, e.g., (II.13) and (II.14) where, using (I.14), $a_{10}=-2 b_{0}^{-1} c_{0} \neq 0$ and $a_{21}=-2 c_{0}$.

We now set

$$
\Gamma_{a s}\left(p_{1} \ldots p_{2 n} ; m^{2}, g\right)=Z(g)^{-n} \Gamma_{0}\left(p_{1} \ldots p_{2 n} ; m^{2}, V(g)\right)
$$

where we have set $U^{2}=m^{2}$ for simplicity, and used that dimensionless parameters can only depend on $g$. Now, from $\Gamma_{a s}$ satisfying the homogeneous PDE (I.12),

$$
\begin{array}{r}
\left\{m^{2}\left[\partial / \partial m^{2}\right]+\beta(g)(d V(g) / d g)[\partial / \partial V]-2 n \gamma(g)-n \beta(g)(d \ln Z(g) / d g)\right\} \\
\left.\cdot \Gamma_{0}\left(p_{1} \ldots p_{2 n} ; m^{2}, V\right)\right|_{V=V(g)}=0 .
\end{array}
$$

Using this for $n=1$ at $p^{2}=-m^{2}$ and for $n=2$ at the corresponding symmetry point yields

and

$$
\beta(g)(d V(g) / d g)=V^{2} R(V)
$$

$$
2 \gamma(g)+\beta(g)(d \ln Z(g) / d g)=V^{2} S(V)
$$

where $R$ and $S$ are power series in $V$. The first relation gives ${ }^{37} V(g)=g P(g)$ and then the second $Z(g)=1+g Q(g)$, with $P$ and $Q$ power series in $g$. Using these expressions in (B.2) gives all functions $\Gamma_{a s}$ in terms ${ }^{38}$ of the $\Gamma_{0}$.

We now show how in the zero-mass theory the singularity structure found in Sections III.1 and III.2 can be displayed such that $\Gamma_{\underline{a s}}$ and $\hat{\Gamma}_{\underline{a s}}$ of those sections can be obtained directly from the zero-mass theory. We use the notation of Appendix A. One easily verifies

$$
\stackrel{0}{\rho^{0}}=H^{0} N^{-1}
$$

37 That this and the following relation do not involve $g \ln g$ follows directly from (B.2), (B.1), and the fact that the $\Gamma_{a s}$ are power series in $g$. The described, complicated construction of $V(g)$ and $Z(g)$ by use of (B.3) avoids, however, the exceedingly complicated determination of the $\Gamma_{a s}$ also at the renormalization points. Actually, with $V(g)$ and $Z(g)$ determined, the renormalization conditions for the theory described by the $\Gamma_{a s}$ can be determined from (B.2) and these functions calculated without need of referring back to the $\Gamma_{0}$.

38 The parametrization (B.1) of the zero-mass theory is closely related to the renormalization conditions used in QED by Gell-Mann and Low [4]. However, the theory (B.1) is a zero-mass theory whose relation to AFs, and not directly to asymptotic behaviour, is given by (II.6) (which e.g. allows to consider corrections, see Section IV.3) and (II.9) rather than by less precise statements. By this two-step-, in the place of the one-step procedure of Ref. [4], statements correct to all orders in perturbation theory (and, e.g. in those statements that rely on (II.4) rather than on (II.5), going beyond it) are separated from assumptions on the behaviour of the zero-mass theory as $g \rightarrow g_{\infty}$, or, equivalently, $V(g) \rightarrow V\left(g_{\infty}\right)=V_{\infty}$. That $V_{\infty}$ is a particular value of $V$ and not just any value is seen from the infinite dilatation implied, and the discussion of Sections IV.1-3 addresses itself to this point. - That there are different types of zero-mass QED, was observed by Baker and Johnson [16]. 
where

$$
\begin{array}{r}
H=\left[1+(1+/ G)(B-\stackrel{0}{B}) G+(1+I G)\left(\stackrel{0}{B}-\stackrel{0}{B}^{0}\right)(G-\stackrel{0}{G})\right]^{-1} \\
\cdot[I-(1+/ G)(B-\stackrel{0}{B})]
\end{array}
$$

and

$$
N=1+<(G-\stackrel{0}{G}) H^{0}
$$

where the $<$ sign means to integrate over the (relative) momentum to its right. In (B.4-6), the total momentum in $H$, and in all kernels $I, G, B$ without 0 on top, is fixed at some nonzero (more precisely, not lightlike) value, and the inverse in (B.5) is defined by expansion. $H$ is $U V$ - and, even in a zero-mass theory, $U R$ convergent, the latter since $\stackrel{0}{B^{p}}-\stackrel{0}{B^{0}}$ vanishes for $p \rightarrow 0$. In contrast, $N$ is $U V$ - but, in a zero-mass theory, not $U R$ convergent. Thus, with total momentum $r\left(r^{2} \neq 0\right)$, and comparing with (II.10),

$$
\begin{aligned}
& \lim _{\lambda \rightarrow 0}\left[a(g(\lambda))^{-2} a(g)^{2}{ }^{p} H^{r}\left(p^{2} m^{-2} \lambda^{-2}, r^{2} m^{-2} \lambda^{-2} ; g(\lambda)\right)\right] \\
& \quad \equiv \bar{H}\left(p^{2} m^{-2}, r^{2} m^{-2} ; g\right)
\end{aligned}
$$

exists and can, in terms of the $\Gamma_{0}$-theory, directly be calculated as

$$
\bar{H}=Z(g)^{-2}{ }^{p}{ }_{H_{0}^{0}}^{0}\left(p^{2}, r^{2} ; m^{2}, V(g)\right)
$$

by virtue of (B.2). Comparing (B.4) with (III.8) and using (B.7) we learn that

$$
\lim _{\lambda \rightarrow 0}\left[h(g(\lambda)) h(g)^{-1} N\left(r^{2} m^{-2} \lambda^{-2} ; g(\lambda)\right)\right] \equiv \bar{N}\left(r^{2} m^{-2} ; g\right)
$$

exists and that

$$
\Gamma_{\underline{a s}}\left(p(-p) 00 ; m^{2}, g\right)=\bar{N}\left(r^{2} m^{-2} ; g\right) \bar{H}\left(p^{2} m^{-2}, r^{2} m^{-2} ; g\right) .
$$

Due to (B.9)

$$
\bar{N}\left(r^{2} m^{-2} ; g\right)=h(g)^{-1} h\left(g\left(\left(-r^{2} / m^{2}\right)^{\frac{1}{2}}\right)\right) \bar{N}\left(-1 ; g\left(\left(-r^{2} / m^{2}\right)^{\frac{1}{2}}\right)\right)
$$

and thus can be calculated if only $\bar{N}(-1 ; g) \equiv v(g)=1+O(g)$ is known, which can be obtained from (B.9), setting $r^{2}=-m^{2}$ by the "elementary recipe" of Section II. Choosing for definiteness $r^{2}=-m^{2}$ in (B.7-10) we have

$$
\Gamma_{\underline{a s}}\left(p(-p) 00 ; m^{2}, g\right)=v(g)^{-1} Z(g)^{-2} p H_{0}^{0}\left(p^{2} ; m^{2}, V(g)\right)
$$

where the evaluation of $H_{0}^{0}$ with $r^{2}=-m^{2}$ is understood. We have not attempted to calculate $v(g)$ in terms of the zero-mass theory (cp. Section IV.3). - We note that, outside of perturbation theory, from (B.9) 
$N\left(-\lambda^{-2} ; g(\lambda)\right) \sim h(g) v(g)\left(b_{0} \ln \lambda^{-2}\right)^{\frac{1}{3}}$ as $\lambda \rightarrow 0$, displaying its $U R$-singularity ${ }^{39}$.

Manipulation of (A.3) and use of (B.4) gives

$$
\stackrel{0}{I}=M\left(r, s ; m^{2}, g\right)-I^{0} N\left({ }^{s} H^{0}\right)^{-1}\left(<(G-\stackrel{0}{G}) H^{s}\right)^{0} I^{0}
$$

where ( $r$ is in each case the total momentum)

with

$$
M\left(r, s ; m^{2}, g\right)=H-H^{0}\left({ }^{s} H^{0}\right)^{-1}\left({ }^{s} H-{ }^{s} K\right)
$$

i.e.

$$
{ }^{p} K^{p^{\prime}}={ }^{p} H^{-p}
$$

$K=[I-(B-\stackrel{0}{B})(1+G /)] \cdot\left[1+G(B-\stackrel{0}{B})(1+G /)+(G-\stackrel{0}{G})\left(\stackrel{0}{B}-{ }^{0} \stackrel{0}{B}\right)(1+G /)\right]^{-1}$

and $s \neq 0$ (more precisely, $s^{2} \neq 0$ ). The two terms in $M(r, s)$ are separately $U V$ - and, in the zero-mass theory, $U R$-convergent. The last term in (B.13a) is $r$ - and $s$-dependent, however, its $U R$-singular part is $r$ - and $s$-independent since (B.13a) is an identity. Comparison of (B.13a) with (III.15) and (III.8) gives

$$
\begin{aligned}
& \Gamma_{\underline{a s}}\left(p(-p) q(-q) ; m^{2}, g\right)=Z(g)^{-2} M_{0}\left(p(-p) q(-q) ; r, s ; m^{2}, V(g)\right) \\
& \quad+i \chi\left(r, s ; m^{2}, g\right) \Gamma_{\underline{a s}}\left(p(-p) 00 ; m^{2}, g\right) \Gamma_{\underline{a s}}\left(q(-q) 00 ; m^{2}, g\right)
\end{aligned}
$$

where

$$
\begin{aligned}
& M_{0}\left(p(-p) q(-q) ; r, s ; m^{2}, V(g)\right) \\
& \quad=Z(g)^{2} \lim _{\lambda \rightarrow 0}\left[a(g)^{2} a(g(\lambda))^{-2}{ }^{2} M^{q}\left(r, s ; m^{2} \lambda^{2}, g(\lambda)\right)\right]
\end{aligned}
$$

is a function obtained in the zero-mass theory (B.1), and

$$
\begin{aligned}
& \chi\left(r, s ; m^{2}, g\right)=\lim _{\lambda \rightarrow 0}\left\{a(g)^{-2} h(g)^{-2}[k(g)-k(g(\lambda))]\right. \\
& \left.\quad+i\left[\Gamma_{\underline{a s}}\left(s(-s) 00 ; m^{2}, g\right)\right]^{-1} h(g)^{-1} h(g(\lambda))\left(<(\stackrel{r}{G}-\stackrel{0}{G}) H^{r}\right)\left(m^{2} \lambda^{2}, g(\lambda)\right)\right\}
\end{aligned}
$$

where the notation means that the last factor is to be evaluated with mass $m \lambda$ and coupling constant $g(\lambda)$, and (III.14) is used. In (B.14b) the limit exists, as in all such formulas in this Appendix, in all orders of perturbation theory such that the "elementary recipe", applied only to the last term in the limitand, is applicable; outside of perturbation theory, the first term in the curly bracket is (see(III.14) and (II.4)) essentially proportional to $\left(\ln \lambda^{-2}\right)^{\frac{1}{3}}$ and so behaves therefore also the second term.

39 That this singularity is not proportional to $\ln \lambda^{-2}$, as (B.6) might suggest, is due to the $U R$ singularity of ${ }^{0} H^{0}$, which arises from ${ }^{0} B^{0}$ then occurring in (B.5). 
We have not attempted to calculate $\chi$ directly in terms of the zero-mass theory.

Finally, we consider the mass vertex of Section III.2. We write it as $\stackrel{0}{\Gamma}^{q}$ and express it with the help of the mass vertex $\Gamma$ at nonzero total momentum as

with

$$
\stackrel{0}{\Gamma}=L-L(G-\stackrel{0}{G})>0 \stackrel{0}{\circ}
$$

$$
\begin{aligned}
L & =\Gamma\left[1+G(B-\stackrel{0}{B})(1+G /)+(G-\stackrel{0}{G})\left(\stackrel{0}{B}-{ }^{0} \stackrel{0}{B}\right)(1+G /)\right]^{-1} \\
& \equiv \Gamma[1+R]^{-1},
\end{aligned}
$$

as is easily obtained by manipulating (A.3). $L$ is $U V$ - but not $U R$-convergent, the $U R$-divergence (for zero mass) being, however, solely due to the one of $\Gamma$ stemming from its imposed normalization $\stackrel{0}{\Gamma}^{0}=1$, see (III.18c). Inserting (B.15a) into (III.21), using (III.18c) and (B.14c) we find easily

$$
\begin{aligned}
& \hat{\Gamma}_{\underline{a s}}\left(q(-q) ; m^{2}, g\right) \\
& =\frac{1}{2}(2 \pi)^{-4} \int d q^{\prime} \hat{\Gamma}_{a s}\left(\left(q^{\prime}+\frac{1}{2} r\right)\left(-q^{\prime}+\frac{1}{2} r\right) ; m^{2}, g\right)[1+R]_{a s}^{-1}\left(q^{\prime}, r, q ; m^{2}, g\right) \\
& \quad+i \Gamma_{\underline{a s}}\left(q(-q) 00 ; m^{2}, g\right) \sigma\left(r^{2} m^{-2}, g\right)
\end{aligned}
$$

with

$$
\begin{aligned}
\sigma\left(r^{2} m^{-2}, g\right)=a(g)^{-2} h(g)^{-2} & \lim _{\lambda \rightarrow 0}\{k(g)-k(g(\lambda)) \\
& \left.+i a(g(\lambda))^{2} h(g(\lambda))^{2}\left[L^{r}\left(\dot{G}^{r}-\stackrel{0}{G}\right)>(m \lambda, g(\lambda))\right]\right\}
\end{aligned}
$$

wherein it is understood that the square bracket is evaluated with mass $m \lambda$ and coupling constant $g(\lambda)$. In (B.16a) it is used that the kernel $R$ does not require any factor for the zero-mass limit in the sense of the theory (B.1) to exist (nor any $Z(g)$-factor: $R_{a s}=R_{0}$ ), and the asymptotic mass vertex at nonzero total momentum is needed. It is from

$$
\Gamma=\Gamma^{0}\left[1-G\left(B-B^{0}\right)\right]^{-1} \equiv \Gamma^{0}[1+S]^{-1}
$$

and (III.18c) calculated as

$$
\stackrel{r}{\Gamma_{a s}^{q}}=\left(\left[1+\stackrel{r}{S}_{a s}\right]^{-1}\right)^{q} \tau\left(r^{2} m^{-2}, g\right)
$$

where

$$
\tau\left(r^{2} m^{-2}, g\right)=\lim _{\lambda \rightarrow 0}\left\{h(g)^{-1} h(g(\lambda)) \Gamma^{r_{0}}\left(m^{2} \lambda^{2}, g(\lambda)\right)\right\} .
$$


In (B.17b), the mass vertex to be inserted is e.g. calculated from

$$
\Gamma^{0}=<\left[1-\stackrel{0}{G}\left(\stackrel{0}{B}-\stackrel{0}{B^{0}}\right)\right]^{-1} \cdot[1+(G-\stackrel{0}{G}) /+\stackrel{0}{G}(B-\stackrel{0}{B})(1+G /)]^{0} .
$$

The point of the relatively complicated formulas (B.14) and (B.16) together with (B.17) is that it suffices to determine the functions $\chi, \sigma$, and $\tau$ for $r^{2}=-m^{2}$ and, e.g., $r=\mathrm{s}$, similarly as we simplified (B.10) to (B.12); their values for arbitrary $r^{2}$ (with, in case, $r=s$ ), if desired, can be obtained from transformation formulas analogous to (B.11).

\section{Appendix C}

\section{Noninfinitesimal Mass Vertex Insertion}

The theory (I.3) can for all $s>-1$ be constructed to arbitrarily high orders in perturbation theory by adaption of a method developped elsewhere (Section V.2 of Ref. [18]) in connection with problems concerning symmetry breaking. That nondifferential method is, however, more complicated than the differential one described here which, although the problem at hand is an academic one, appears to be instructive.

We compare (I.5) with (I.12) and find, using (I.14a),

and

$$
\begin{gathered}
d g(s) / d \ln m^{2}(s)=\beta(g(s)) \\
d \ln Z(s) / d \ln m^{2}(s)=2 \gamma(g(s))
\end{gathered}
$$

$$
\begin{aligned}
& \Delta \Gamma\left(p_{1} \ldots p_{2 n} ; m^{2}(s), g(s)\right) \\
& \quad=Z(s)^{n}\left(d \ln m^{2}(s) / d s\right)^{-1}[\partial / \partial s] \Gamma^{s}\left(p_{1} \ldots p_{2 n} ; m^{2}, g\right) .
\end{aligned}
$$

We set $n=1, p_{1}=-p_{2}=0^{40}$ and obtain, using (I.8),

$$
\begin{aligned}
& \Delta \Gamma\left(00 ; m^{2}(s), g(s)\right) \\
& \quad=Z(s)\left(d \ln m^{2}(s) / d s\right)^{-1}[\partial / \partial s] \Gamma^{s}\left(00 ; m^{2}, g\right)=-i m^{2}(s) \varphi(g(s)) .
\end{aligned}
$$

Since, using (I.3), techniques of Ref. [8], (C.3), and (III.4),

$$
\begin{aligned}
{\left[\partial^{2} / \partial s^{2}\right] \Gamma^{s}\left(00 ; m^{2}, g\right) } & \\
= & Z(s)^{-2}\left(d \ln m^{2}(s) / d s\right)^{2}\left[-\frac{1}{2} i m^{2}(s) \varphi(g(s))\right]^{2} \\
& \cdot \iint d x d y\left\langle T N_{2}\left\{\phi(x)^{2}\right\} N_{2}\left\{\phi(y)^{2}\right\} \tilde{\phi}(0) \tilde{\phi}(0)\right\rangle^{s \text { prop }} \\
= & -i Z(s)^{-2}\left(d \ln m^{2}(s) / d s\right)^{2} m^{2}(s) \varphi(g(s)) \eta(g(s)) \\
= & -i[d / d s]\left[Z(s)^{-1}\left(d \ln m^{2}(s) / d s\right) m^{2}(s) \varphi(g(s))\right] \\
\equiv & -i[d / d s] u(s)
\end{aligned}
$$

40 The reason for this choice rather than, e.g., $p_{1}{ }^{2}=p_{2}{ }^{2}=m^{2}$, is that the necessary calculations were already performed in Section III. 
we have

$$
d u(s) / d \ln m^{2}(s)=Z(s)^{-1} \eta(g(s)) u(s)
$$

wherefrom

$$
\ln u(s)=\int_{m^{2}}^{m^{2}(s)} m^{2}\left(s^{\prime}\right)^{-1} d m^{2}\left(s^{\prime}\right) Z\left(s^{\prime}\right)^{-1} \eta\left(g\left(s^{\prime}\right)\right)+\text { const. }
$$

The scale of $s$ is fixed by the convention (I.7), see (I.13a) and (I.14a), as

$$
d \ln m^{2}(s) /\left.d s\right|_{s=0}=1
$$

which yields the const in (C.5). Thus

$$
\begin{aligned}
& d \ln m^{2}(s) / d s \\
& \quad=m^{2} \varphi(g) Z(s) m^{2}(s)^{-1} \varphi(g(s))^{-1} \exp [\text { the integral in (C.5)] }
\end{aligned}
$$

and, finally,

$$
\begin{aligned}
& s=m^{-2} \varphi(g)^{-1} \int_{m^{2}}^{m^{2}(s)} d m^{2}\left(s^{\prime}\right) Z\left(s^{\prime}\right)^{-1} \varphi\left(g\left(s^{\prime}\right)\right) . \\
& \cdot \exp \left[-\int_{m^{2}}^{m^{2}\left(s^{\prime}\right)} m^{2}\left(s^{\prime \prime}\right)^{-1} d m^{2}\left(s^{\prime \prime}\right) Z\left(s^{\prime \prime}\right)^{-1} \eta\left(g\left(s^{\prime \prime}\right)\right)\right] .
\end{aligned}
$$

Using in the integrands the solutions of the differential Eqs. (C.1) as a function of $\mathrm{m}^{2}(s)$, or, more conveniently, of $g(s)$, gives $s$, and, by inversion, all functions $m^{2}(s), g(s)$, and $Z(s)$, can ultimately be obtained as functions of $s$, to arbitrarily high order in $g$.

(C.7) gives with (C.1) and (C.6)

$$
d^{2} \ln m^{2}(s) /\left.(d s)^{2}\right|_{s=0}=2 \gamma(g)-\varphi(g)^{-1} \varphi^{\prime}(g) \beta(g)+\eta(g)-1 \equiv f(g)-1
$$

wherefrom

$$
m^{2}(s)=m^{2}\left[1+s+\frac{1}{2} s^{2} f(g)+\ldots\right] .
$$

That $f(g)$ is $O(g)$ from (III.4) comes from the fact that $L$ in (I.1) involves in $\delta m^{2}$ a term $\propto g G(00)$ usually hidden by the use of Wick contradictions; since such contractions, however, depend on the bare (or, alternatively, the true) mass, if one keeps the contraction fixed but changes the bare mass, an effect in order $g$ arises.

\section{References}

1. Weinberg, S.: Phys. Rev. 118, 838 (1960).

2. Fink, J. P.: J. Math. Phys. 9, 1389 (1968).

3. Westwater, M. J.: Fortschr. d. Phys. 17, 1 (1969).

4. Gell-Mann, M., Low, F.E.: Phys. Rev. 95, 1300 (1954). 
5. Bogoliubov, N.N., Shirkov,D.V.: Introduction to the Theory of Quantized Fields. New York: Interscience Publ. 1959.

6. Landau,L.D., Abrikosov,A., Khalatnikov,I.: Nuovo Cimento Suppl. 3, 80 (1956).

7. Appelquist, T., Primack, J.R.: Phys. Rev. 1D, 1144 (1970).

8. Symanzik, K.: Commun. math. Phys. 18, 227 (1970).

9. Wilson, K. G.: Phys. Rev. 179, 1499 (1969).

10. Symanzik, K.: In: Springer Tracts in Modern Physics Vol. 57, (1971), p. 222.

11. Wilson, K.G.: preprint, Cornell U., 1964.

12. Brandt, R.A.: Ann. Phys. 44, 221 (1967).

13. Zimmermann, W.: In: Lectures on Elementary Particles and Fields (1970 Brandeis University Summer Institute), Vol. 2. Eds.: Deser,S., Grisarv, M., Pendleton, H. Cambridge, Mass.: MIT Press 1971.

14. Coleman, R., Jackiw, R.: Ann. Phys. (to appear).

15. Wilson, K. G.: Phys. Rev. P. 3, 1818 (1971).

16. Baker, M., Johnson, K.: Phys. Rev. D 3, 2516, 2541 (1971).

17. Symanzik, K.: Commun. math. Phys. 16, 48 (1970).

18. - In: Cargèse Lectures in Physics, Vol. 6. Ed. J. D. Bessis. New York: Gordon and Breach 1971.

19. Wu, T.T.: Phys. Rev. 125, 1436 (1962).

20. Johnson, R. W.: J. Math. Phys. 11, 2161 (1970).

21. Callan Jr., C. G.: Phys. Rev. D 2, 1541 (1970).

22. Kinoshita, T.: J. Math. Phys. 3, 650 (1962).

23. Ruelle,D.: Nuovo Cimento 19, 356 (1961).

24. Schroer, B.: Fortschr. d. Phys. 11, 1 (1963).

25. Landau, L. D.: In: Niels Bohr and the developments of Physics, ed. W. Pauli, New York: McGraw Hill Book Co. 1955; and In: Physics in the Twentieth Century, eds. R. Jost, V.Weisskopf.

26. Pohlmeyer, K.: Commun. math. Phys. 12, 204 (1969).

27. e.g., Fritzsch,H., Gell-Mann, M., CALT-68-297.

28. Lehmann, H.: Nuovo Cimento 11, 342 (1954).

29. Symanzik, K.: In: Lectures on High Energy Physics, ed. B. Jakśič, Zagreb: 1961. New York: Gordon and Breach 1965.

30. Taylor, J. G.: Suppl. al Nuovo Cimento 1, 857 (1963).

31. Ferretti, B.: Nuovo Cimento (L) 12, 457 (1954).

32. Hahn, Y., Zimmermann, W.: Commun. math. Phys. 10, 330 (1968); Zimmermann, W.: Ibid. 11, 1 (1968).

33. Hepp, K.: Théorie de la renormalisation. Berlin-Heidelberg-New York: Springer 1969.

\author{
K. Symanzik \\ Deutsches Elektronen-Synchrotron \\ BRD-2000 Hamburg 52 \\ Notkestieg 1
}

